\title{
Prasiolales (Chlorophyta) von Helgoland
}

\author{
P. Kornmann \& P.-H. SahLING \\ Biologische Anstalt Helgoland (Meeresstation); \\ Helgoland, Bundesrepublik Deutschland
}

\begin{abstract}
Prasiolales (Chlorophyta) of Helgoland. In their natural habitats, some representatives of Prasiolales are regularly, or often, washed by waves; others are sprayed with sea-water only occasionally, or not at all. In correspondance to this ecological grouping, species require marine or freshwater media respectively for optimal growth in cultures. The halophilic group consists of Prasiola stipitata and Rosenvingiella constricta. Though not analysed in detail, morphological characters and behaviour in cultures suggest that several different species are united under the name Prasiola stipitata. Rosenvingiella constricta, new for Helgoland and thus far reported only twice in Europe, is a dioecious haplont; both meiosis and genotypic sex-determination occur in the germinating zygote. The parenchymatous "Gayella-stage" of Rosenvingiella polyrbiza proved to be male and female gametangia, producing spermatozoids and non-motile eggs respectively. Fertile threads were extremely rare in natural material and - contrary to Rosenvingiella constricta - did not occur in cultures, so the life cycle could not be elucidated. Prasiola calophylla is frequently associated with Rosenvingiella polyrhiza. The life cycle includes heteromorphic asexual generations, the spores of the leafy thallus giving rise to unicellular aplanosporangia. Prasiola furfuracea reproduces only by spores. Samples of Rosenvingiella polyrbiza frequently contain Prasiolopsis in addition to a number of other Chlorophyceae. Consequently, it is easy to appreciate that previous investigators came to erroneous conclusions about life histories and biological relations in studies based only on field material.
\end{abstract}

\section{EINLEITUNG}

Als diese Studie mit der Beobachtung der Geschlechtspflanzen von Rosenvingiella polyrbiza im Februar 1973 begann, war nicht vorauszusehen, daß sie sich so schnell zu einer umfassenden Bearbeitung der bei Helgoland vorkommenden Vertreter der Prasiolales ausweiten sollte. Ganz von selbst ergab sich die entwicklungsgeschichtliche Untersuchung einer ziemlich regelmäßig in den Watten von Rosenvingiella vorkommenden zarten Prasiola, deren Identität mit $P$. calophylla wahrscheinlich ist.

Bei den regelmäßigen Kontrollen der Fundplätze von Rosenvingiella wurde im Juli 1973 Prasiola furfuracea auf einer kleinen Fläche gesammelt. Auch sie wurde in Nährlösung fertil, ihre Entwicklung konnte im Kulturexperiment vollständig verfolgt werden. Die dabei gewonnenen Ergebnisse stehen nicht mit denen von WILLE (1906) in Einklang. Mit dem Ausgangsmaterial gelangte neben zahlreichen nicht näher untersuchten mikroskopischen Grünalgen auch Prasiolopsis ramosa in die Kulturen. 
Alle bisher genannten Algen leben auf Hafenmolen und Uferbefestigungen, an denen sie nicht vom Seewasser erreicht, wohl aber gelegentlich durch Spritzwasser benetzt werden. Sie gedeihen gut in Nährlösungen, die mit Süßwasser bereitet sind, in denen sie dauernd untergetaucht wachsen. Die "atmophytische" Lebensweise am natürlichen Standort wird also mehr ertragen als sie eine Lebensnotwendigkeit ist. Daher ist es auch weder erforderlich noch zweckmäßig, in den Kulturen die Bedingungen des natürlichen Standorts nachahmen zu wollen.

Die in die stipitata-Gruppe einzuordnenden Prasiola-Arten werden dagegen täglich von Seewasser überflutet oder erhalten häufig Spritzwasser. Sie bevorzugen in der Kultur Seewasser-Nährlösungen; in ihnen werden sie erst nach üppigem Wachstum fertil, während sie in Nährlösungen mit Süßwasser wesentlich kleiner bleiben und frühzeitig fertil werden. Auf den Hafenmolen können solche halophilen Prasiola-Arten vereinzelt auch zwischen Rosenvingiella vorkommen; sie werden durch vergleichende Kulturen in beiderlei Medien leicht als solche erkannt. Es war nicht beabsichtigt, den stipitata-Komplex näher zu analysieren; die Beobachtungen am Standort und das Ergebnis zahlreicher Kulturversuche lassen jedoch darauf schließen, daß Prasiola ,stipitata" mehrere selbständige Arten einschließt. Damit ist auch die Grundlage unsicher geworden, auf der Friedmann (1969) Lebenszyklus und Morphologie von Prasiola "stipitata" zu geographischen und Umweltfaktoren in Beziehung zu bringen versucht. Geschlechtspflanzen von Prasiola "stipitata" sind bei Helgoland noch nicht gefunden worden.

Der überraschendste Fund war Rosenvingiella constricta. Sie teilt den Standort mit Prasiola "stipitata" und gedeiht wie diese am besten in Erdschreiberlösung. Der vollständige Lebenszyklus konnte im Kulturversuch beobachtet werden. Die so oft - zuletzt von FrIEDmanN (1969) - geäußerte Vermutung einer genetischen Beziehung zwischen Rosenvingiella und Prasiola muß endgültig aufgegeben werden. Ob die jetzt bei Helgoland entdeckte und bisher nur zweimal in Europa gefundene Rosenvingiella constricta (WAERN 1952) mit dem von der kalifornischen Küste stammenden Typus übereinstimmt, bleibt eine offene Frage.

\section{ERGEBNISSE}

\section{Rosenvingiella constricta (Setchell \& Gardner) SIlva}

\section{Vorkommen und Morphologie}

Auf dem isolierten und nur mit einem Boot erreichbaren Abschnitt der Helgoländer Nordmole wurde Rosenvingiella constricta erstmalig am 7. September 1973 gefunden. Es waren nur einzelne kleine Stellen auf der stark von Prasiola stipitata besiedelten Molenfläche, die durch ihr andersartiges Aussehen auffielen. In Abbildung 1 A-C sind mehrere Proben der Alge dargestellt; mehr oder weniger lange monosiphone Basalabschnitte verbreitern sich zu unregelmäßig eingeschnürten polysiphonen Keulen. Die einzelnen Fäden sind zu kleinen Büscheln vereinigt und überziehen das Substrat rasenartig. Mikrotomschnitte durch lebend eingefrorenes Material zeigen den Aufbau 


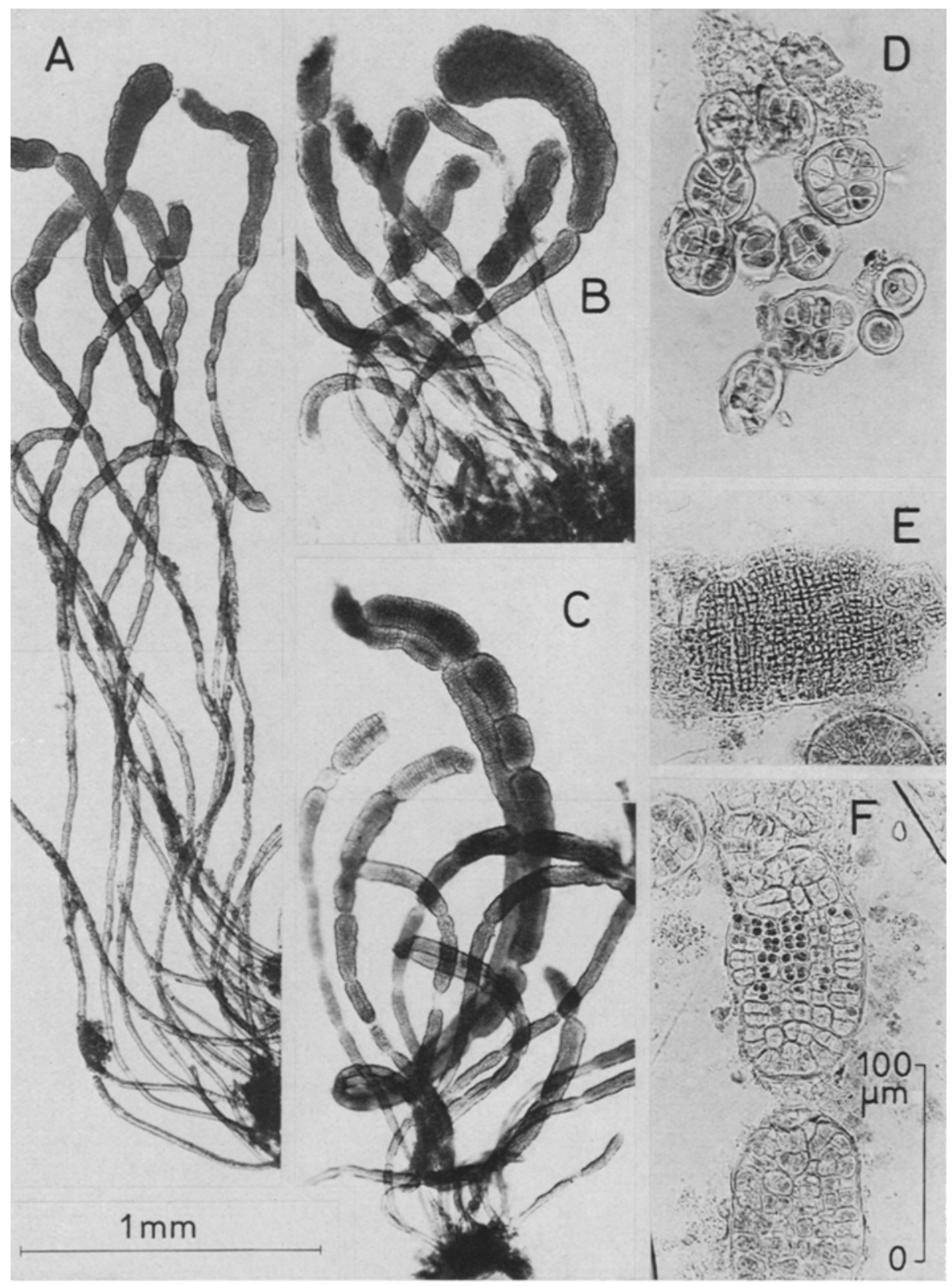

Abb. 1: Rosenvingiella constricia. Helgoland, 7.9.1973. A-C Variabilität des Naturmaterials. $D$ Querschnitte durch monosiphone und parenchymatische Fadenabschnitte. $E, F$ Reife männliche und weibliche Thalli im Längsschnitt. $D-F$ sind Schnitte durch lebend eingefrorenes Material 


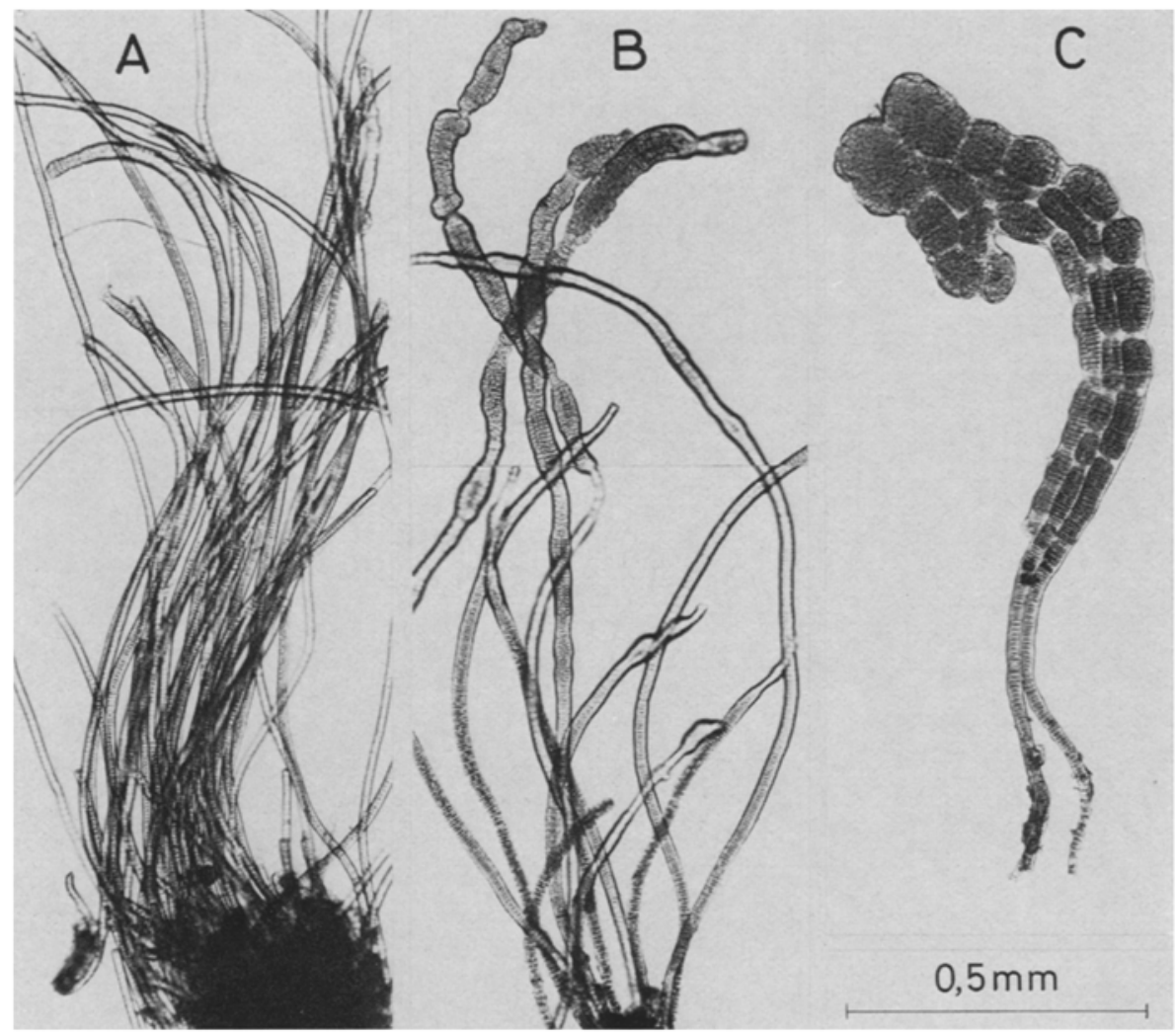

Abb 2: Rosenvingiella constricta. Helgoland, 1, 10.1973. A-C Variabilität des Naturmaterials

und die Entstehung des polysiphonen Fadens (Abb, 1D). Die zahlreichen fertilen Pflanzen ließen sich ohne weiteres ihrem Geschlecht zuordnen; die männlichen Pflanzen waren an den kleingefächerten, hell- bis olivgrün gefärbten Keulen leicht von den großfächerigen dunkelgrünen weiblichen Pflanzen zu unterscheiden. Entsprechende Längsschnitte gibt Abbildung $1 \mathrm{E}, \mathrm{F}$ wieder, aus den meisten angeschnittenen Zellen sind die reifen Eier herausgefallen.

In einer etwa drei Wochen später, am 1. Oktober 1973, gesammelten Probe war der Habitus etwas abgeändert. Wohl waren auch noch Büschel der gedrungenen, ausgewachsenen und fertilen Pflanzen vorhanden, wie auch die häufiger vorkommende Verwachsung mehrerer Thalli (Abb. $2 \mathrm{C}$ ). Bemerkenswert waren aber die Büschel aus verhältnismäßig langen monosiphonen Fäden, deren apikale Enden gerade erst mehrreihig wurden (Abb. $2 \mathrm{~A}$ ). In anderen, gleichhohen Pflanzen war die parenchymatische Differenzierung basal weiter fortgeschritten (Abb. 2 B). Die Abbildungen 1 und 2 zeigen die Variation der Erscheinungsform von Rosenvingiella constricta in ihrer altersmäßigen Bedingtheit.

In ihrem Aussehen stimmen die Pflanzen von Helgoland gut mit WAERNs Zeich- 
nungen des kalifornischen Cotypus und einer Probe aus dem Skagerrak (leg. Du RIETz 15.7. 1917) überein (1952, Fig. 20, f-j). WAERN hat Rosenvingiella constricta auch in einer Aufsammlung von Gotland, Ostsee (leg. Sernander 4. 7. 1916) gefunden. Seine Zeichnungen sind eine willkommene und wertvolle Ergänzung der Originalbeschreibung (SETCHELl \& GARDNER in GARDNER 1917).

\section{Entwickling}

Das am 7. September 1973 nach gründlichem Auswaschen mit Seewasser in Erdschreiberlösung übertragene Material entleerte bei $15^{\circ} \mathrm{C}$ drei Stunden später spontan große Mengen von Geschlechtszellen. Kurze Zeit nach dem Austreten der Eier schwärmten die Spermatozoiden aus. Die Beobachtung der Kopulation war daher besonders eindrucksvoll, wenn eine sich ausbreitende Wolke der nicht phototaktischen Spermatozoiden die auf dem Boden der Schale liegenden Eier erreichte. Nach der Anheftung der Spermatozoiden gerieten die Eier zunächst in unregelmäßige Bewegung, nach wenigen Augenblicken schwammen die gedrungen-birnförmigen Kopulanten in der Flüssigkeit, durch die am Hinterende befindliche nicht verschmolzene Geißel des Spermatozoids angetrieben. Nur wenige Minuten dauerte es, bis die befruchteten Eier wieder ruhig am Boden lagen. Ganz entsprechende Beobachtungen machten auch Fujryama (1955) und Friedmann (1960) bei der Befruchtung der Eier von Prasiola japonica und Prasiola stipitata.

Die Gametenentleerung ließ sich auch an den beiden folgenden Tagen durch die gleiche Behandlung des Materials - Ausspritzen auf einem Gazesieb mit Seewasser und ruhiges Stehenlassen in Erdschreiberlösung bei $15^{\circ} \mathrm{C}$ - erzielen. Nur einmal konnte der Vorgang des Austritts von Eiern direkt beobachtet werden. Durch eine seitliche Offnung in einem von zwei Einschnürungen begrenzten fertil gewordenen Thallusabschnitt gelangte der größte Teil der Eier ins Freie. Sie strömten ganz gleichmäßig hintereinander auf einer etwas gewundenen „Straße" zum Ausgang, ein Bild, das an die Entleerung eines plurilokulären Ectocarpus-Sporangiums erinnerte.

Lebende Eier (Abb. $3 \mathrm{~A}$ ) sind 4,5 bis $6 \mu \mathrm{m}$ dick; die gleich nach dem Fixieren und Anfärben mit sehr verdünntem Hämatoxylin nach EHRLICH photographierten Spermatozoiden sind 4 bis $5 \mu \mathrm{m}$ lang und ziemlich gedrungen, das spitze Vorderende trägt zwei Geißeln von etwa doppelter Körperlänge (Abb. 3 B, zusammen mit einem Ei und einer Verschmelzung).

Während der ersten Woche ihrer Entwicklung zeigen die Zygoten keine Besonderheiten, sie werden dicker und zum Teil auch etwas elliptisch (Abb. 3 C). Dann wird ihr Inhalt durch zwei aufeinanderfolgende kreuzweise Teilungen in Quadranten zerlegt, und die so entstandenen Zellen wölben sich an einem Ende rhizoidartig vor. Eine 11 Tage alte Kultur enthält alle Stadien dieses Entwicklungsvorganges, den die Zygotenkeimlinge verschieden schnell durchlaufen (Abb. $3 \mathrm{D}$ ). Die Rhizoide verlängern sich und werden durch eine Querwand von den apikalen Zellen getrennt, die sich unmittelbar zu einreihigen Fäden entwickeln können (Abb. 3 E-G). In den meisten Fällen jedoch bilden die vier Schwesterkeimlinge frühzeitig apikal ebenfalls ein Rhizoid aus, so daß die jungen Fäden an beiden Seiten verankert werden und zu Fadenschleifen 


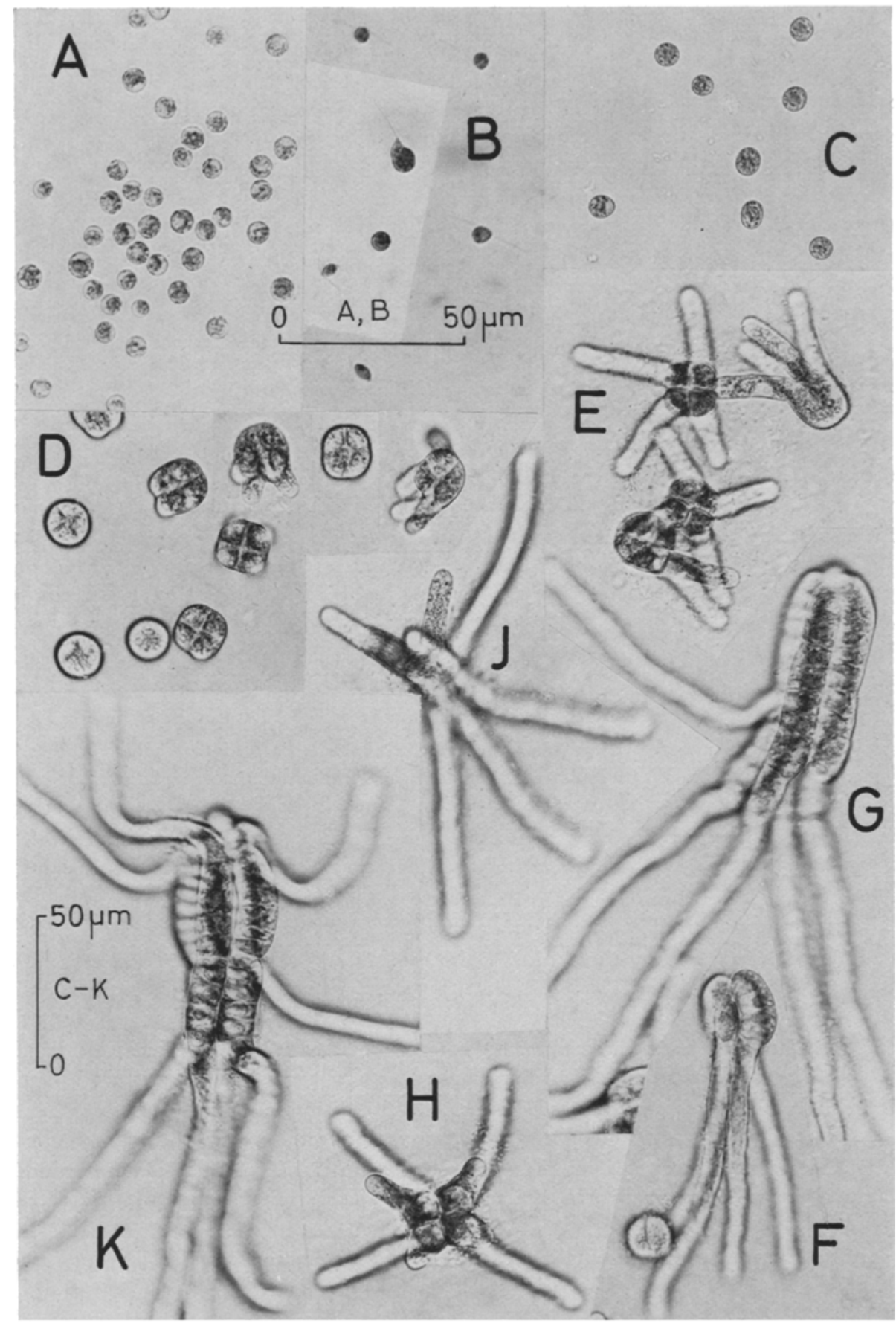

Abb. 3: Rosenvingiella constricta. A Frisch entleerte Eier, lebend. B Spermatozoiden, ein Ei und eine bewegliche Zygote, fixiert mit stark verdünntem Hämatoxylin nach EHRLICH. $C 5$ Tage alte Zygoten. $D 11$ Tage alte Kultur keimender Zygoten. $E-G$ Entwicklung zu Schwesterfäden mit basalem Rhizoid, $H-K$ mit Rhizoiden an beiden Enden 

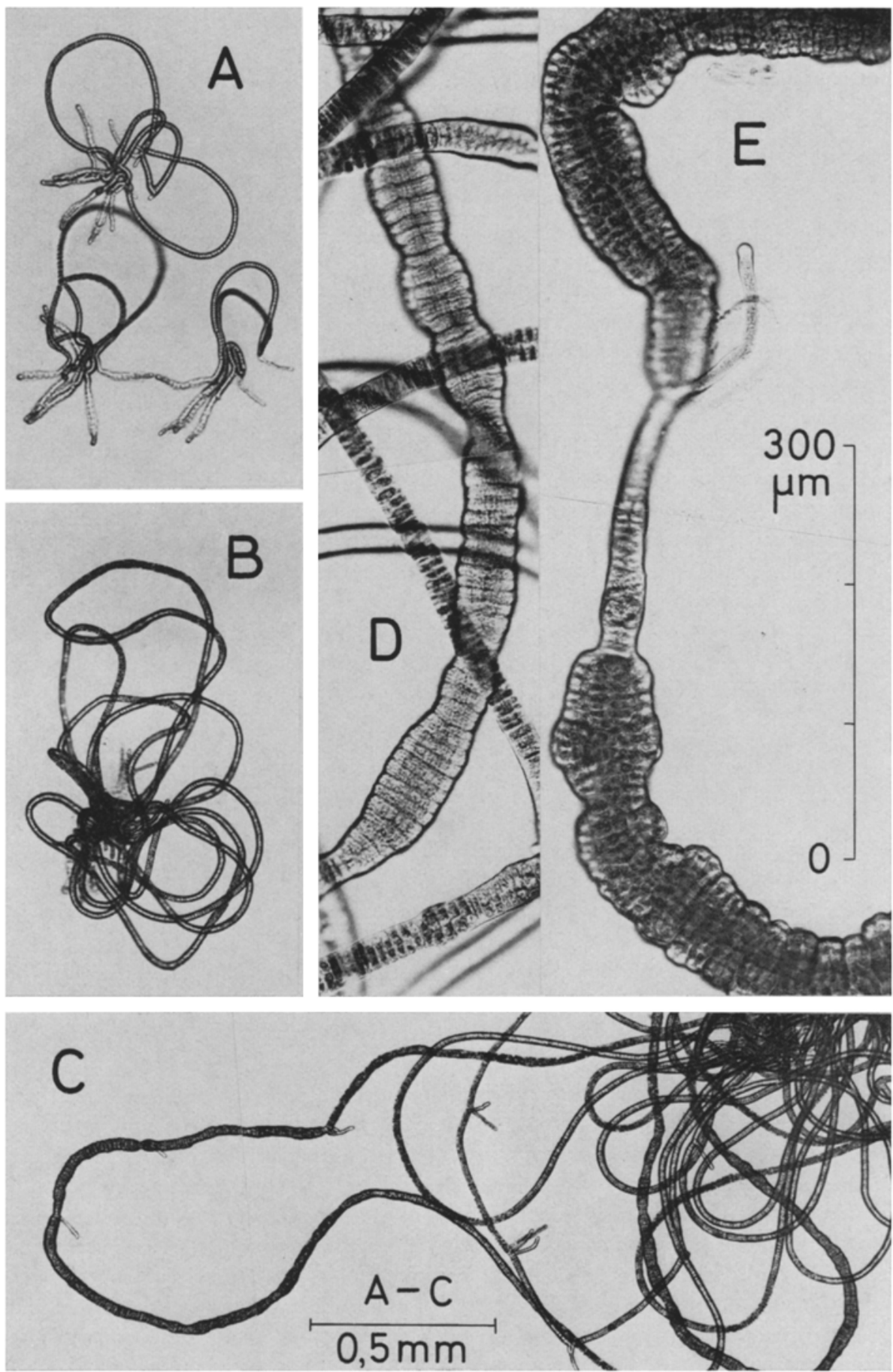

Abb. 4: Rosenvingiella constricta. Aus Zygoten kultivierte Generation: $A 31$ Tage alt, $B 41$ Tage alt mit ersten Längsteilungen, $C$ fertil, 7 Wochen alt. $D, E$ Männliche und weibliche Fadenabschnitte 
auswachsen (Abb. $3 \mathrm{H}-\mathrm{K}$ ). Früher oder später trennen sich aber auch die zunächst polar differenzierten, an der Spitze lose vereinigten Keimlinge voneinander, Rhizoide können apikal oder aus Fadenzellen gebildet werden. Das Ergebnis sind schließlich in jedem Falle Fadenschleifen; die in Abbildung $4 \mathrm{~A}$ dargestellten Pflanzen sind 31 Tage alt.

Frithzeitig isolierte Fadenknäuel wuchsen rasch heran; in 41 Tage alten Kulturen wurden die ersten Längswände in den Fäden beobachtet (Abb. $4 \mathrm{~B}$ ), bereits eine Woche später reife Gametangien (Abb. 4 C-E). Die Nachkommenschaft einer Zygote enthielt männliche und weibliche Fäden. Die Gametenentleerung erfolgte spontan, und bald entwickelten sich in allen Kulturschalen die Keimlinge einer neuen Generation. Die entleerten Fadenabschnitte reißen leicht durch mechanische Wirkung der kräftig wachsenden vegetativen Fäden auseinander.

Die Fähigkeit der Eier zur parthenogenetischen Entwicklung ist außerordentlich gering und dürfte nach grober Schätzung $1 \%$ nicht überschreiten. Unbefruchtete Eier keimen nur langsam und unregelmäßig; im günstigsten Falle entsteht unmittelbar ein monosiphoner Faden, basal oder an beiden Enden mit Rhizoiden. Oftmals differenziert sich auch ein Faden erst nach längerer Zeit aus einem Zellkomplex. Im Vergleich zu der ganz gleichmäßigen und raschen Keimung der Zygoten gewinnt man den Eindruck, daß die parthenogenetische Entwicklung überhaupt nur unter den günstigen Bedingungen der Kultur möglich ist. Ungeschlechtliche Fortpflanzungsorgane oder -zellen wurden bei Rosenvingiella constricta nicht beobachtet.

\section{Allgemeine Betrachtungen}

Die Aufeinanderfolge geschlechtlicher Generationen und die Entstehung von vier gleichartigen Fäden aus der keimenden Zygote lassen vermuten, daß Rosenvingiella constricta ein Haplont und nur die Zygote diploid ist. Diese Annahme ließ sich im Kulturexperiment durch eine "Tetradenanalyse" bestätigen, indem die vier Fadenschleifen geeigneter Pflänzchen getrennt aufgezogen wurden. Das Ergebnis war eindeutig: zehn Analysen ergaben jeweils zur Hälfte männliche und weibliche Pflanzen. Mit der Reduktionsteilung in der keimenden Zygote erfolgt die genotypische Geschlechtsbestimmung.

Ein entsprechender zygotischer Kernphasenwechsel wurde von Fujryama (1955) für die monözische Prasiola japonica zytologisch nachgewiesen. Die Keimungsstadien der Zygote sind denen von Rosenvingiella constricta sehr ähnlich. Meist wachsen drei Zellen zu Rhizoiden aus, während die vierte sich vergrößert und dem flächigen Thallus den Ursprung gibt. Er wird mosaikartig fertilisiert wie Prasiola stipitata und P. meridionalis. Diese beiden Arten sind jedoch Diplohaplonten mit einheitlichem Thallus; die vegetative diploide Basis trägt die fertile haploide Zone (FrIEDMANN 1959 beziehungsweise Cole \& Akintobi 1962). In diesem Zusammenhang ist es bemerkenswert, daß die Zygoten dieser beiden Arten auch in ganz anderer Weise keimen als die von Prasiola japonica: aus ihnen entsteht unmittelbar ein monosiphoner Keimling (FrIEDMANN 1960, Cole \& Axintobi 1962).

Keine Beziehung läßt sich zu den Ergebnissen der Untersuchungen von BRAvo 
(1962, 1965) knüpfen. Die Autorin erhielt in ihren Kulturen von Prasiola meridionalis ein Rosenvingiella-Stadium als randständigen oder der Basis des Thallus entsprossenden Auswudhs. Tatsächlich gleicht Abbildung 4 ihrer Arbeit von 1962 völlig Rosenvingiella constricta, so daß BRAvo sie für eine Erscheinungsform von Prasiola meridionalis hält, mit der sie übrigens den Standort teilt. Allerdings sind die abgebildeten Rosenvingiella-Stadien getrenntgeschlechtlich (BRavo 1965, Fig. 5, 6), während der Thallus von Prasiola meridionalis monözisch ist. Und schließlich ist ihr Rosenvingiella-Stadium vor der Gametenbildung diploid wie der flächige Thallus. Eine Klärung dieser eigenartigen Zusammenhänge dürfte wohl nur durch das Kulturexperiment mit Rosenvingiella constricta vom Standort des Typus zu erwarten sein.

Die in Kultur entstandenen Fadenknäuel von Rosenvingiella constricta sind in ihrem Habitus von den aufrechten rasenartigen Büscheln am natürlichen Standort sehr verschieden. Das ist verständlich, weil sie ja nicht wie diese der mechanischen Wirkung durch ïberrollende oder aufschlagende Wellen ausgesetzt sind. Indessen rechtfertigt die Wuchsform der kultivierten Pflanze eigentlich erst ihre Zuordnung zu Rosenvingiella, während die Erscheinungsform des Naturmaterials seine Eingliederung in diese Gattung beinahe als glücklichen Zufall erscheinen läßt. Der monosiphone Anteil des Thallus mit Rhizoiden tritt hier stark zurück. Ein vegetativer parenchymatischer Thallus existiert eigentlich nicht, denn mit der Längsteilung der Fäden wird die Gametangienbildung eingeleitet. Die keuligen eingeschnürten Gametangien sind für Rosenvingiella constricta charakteristisch, während bei $R$. polyrhiza das Fadenstadium bei weitem überwiegt und parenchymatische fertile Abschnitte nur selten gefunden werden.

\section{Rosenvingiella polyrbiza (ROSENVINGE) SILVA}

\section{Die Gameten}

Ganz im Gegensatz zu Rosenvingiella constricta, deren Lebenszyklus sich binnen zwei Monaten im Kulturexperiment lückenlos klären ließ, bleiben die Zusammenhänge bei Rosenvingiella polyrbiza noch weitgehend unbekannt. Das liegt einerseits daran, daß sie in den Kulturen niemals fertil wurde. Zum anderen waren fertile Fadenabschnitte in dem im Frühjahr 1973 gesammelten Material nur äußerst selten zu finden; es bedurte mehrstündigen Suchens, um einige wenige solcher Stücke zu erhalten. Außerdem gingen häufig die parenchymatischen Fadenabschnitte in den Kulturen durch bakterielle Zersetzung zugrunde, während die angrenzenden monosiphonen Teile nicht geschädigt wurden und weiterwuchsen. Kam es dann doch gelegentlich zur Gametenentleerung, so fehlte das andere Geschlecht, so daß niemals Zygoten erhalten werden konnten. Abbildung 5 zeigt bei A einen weiblichen Faden, der einen Teil der Eier entleert hat $\left(A_{1}\right)$. Nur wenige der unbefruchteten Eier von 5 bis $6,5 \mu \mathrm{m}$ Durchmesser können sich parthenogenetisch entwickeln; auf die unterschiedliche Breite der so entstandenen Fäden sei hingewiesen (C).

Das Ausschwärmen eines männlichen Fadens konnte nur einmal in so eindrucksvoller Weise beobachtet werden, wie Abbildung $5 \mathrm{~B}$ wiedergibt. Der nahezu vollstän- 
dig entleerte Fadenabschnitt war in eine Wolke von schwach beweglichen Spermatozoiden eingehüllt. Die Spermatozoiden $\left(B_{1}\right)$ waren im Durchschnitt $3 \mu \mathrm{m}$ lang, am zugespitzten Vorderende mit zwei gleichlangen Geißeln von etwa doppelter Körperlänge versehen (Maßangaben nach dem einzigen vorhandenen Photo).

Die von Januar bis Ende April 1974 wiederholten Beobachtungen erbrachten leider keine neuen Ergebnisse. Parenchymatische Fadenabschnitte waren etwas reichlicher als im Vorjahr zu finden, jedoch wurden niemals Gameten entleert, weder von dem in Nährlösung gehaltenen noch von dem regelmäßig am natürlichen Standort gesammelten Material. Im Januar und Februar wuchsen die Zellen der parenchymatischen Abschnitte in den Rohkulturen im allgemeinen zu einem dichten Pelz von monosiphonen Fäden aus; später verblaßten sie hier ebenso wie in dem Naturmaterial und gingen zugrunde. Die im Frühjahr 1973 beobachtete Reifung und Entleerung der Gameten war also ein durch besondere Umstände begünstigter glücklicher Zufall.

\section{Vorkommen und Morpbologie}

Rosenvingiella polyrbiza ist auf dem Beton der Molen und Uferbefestigungen von Helgoland wie auch Blidingia marginata f. marginata eine der gemeinsten Algen; die im trockenen Zustand gelbgrünen Watten und Flecke lassen sich leicht als Rosenvingiella ansprechen, sie werden bei Benetzung sofort dunkelgrün. Wir haben dieser schon kaum mehr marinen Vegetation bisher wenig Beachtung geschenkt, und es wäre wohl auch bei einer bloßen Erwähnung des Namens geblieben, wenn nicht der Fund von Geschlechtspflanzen eine eingehendere Untersudhung veranlaßt hätte. Auch innerhalb der Ortschaft ist Rosenvingiella polyrbiza weit verbreitet.

Ein Vergleich der zahlreichen gesammelten Proben ließ eine erhebliche Variabilität ihrer äußeren Erscheinungsform erkennen. Es war daher zu prüfen, ob nicht verschiedene Formen unterschieden werden müssen, weisen doch auch die Angaben in der Literatur in die gleiche Richtung. Rosenvinges Typusmaterial von Grönland besteht aus 11-12 $\mu \mathrm{m}$ breiten Fäden mit scheibenförmigen Zellen (1893, Fig. 45 E-G). Der Habitus der von WiLle (1901) im Oslofjord gesammelten Alge ist jedoch ein ganz anderer. Hier sind die Zellen nicht scheibenförmig, sondern im ausgewachsenen Zustand ebenso lang wie breit. Wenn auch im Text die Fadenbreite mit 10-12 $\mu \mathrm{m}$ angegeben wird, so übersteigt sie in seinen Abbildungen $9 \mu \mathrm{m}$ nicht (Tafel I, Fig. 41-44). BørgEsENs Material von den Färöer (1902) enthielt offenbar beide Formen. "They consist originally and for the most part of cell-filaments (about 10-12 $\mu$ thick) formed of disc-shaped cells which are generally only about one-third as long as broad, though they are sometimes met with as long as they are broad" (p. 482). Wie aus seinen Abbildungen klar zu ersehen ist, haben die dünneren Fäden isodiametrische Zellen (Fig. $99 \mathrm{c}$, $e, h)$, während der breite Faden bei $k$ scheibenförmige Zellen hat.

Schließlich sind auch die präzisen Maßangaben und Zeichnungen bei WAERN (1952) recht aufschlußreich. "The threads at Oregrund and at the southern Alland Sea only measured maximum $10 \mu$ in breadth, while the uniseriate threads of Bohuslän measured $-11-19 \mu$ in breadth, being also much longer" (p. 47). Die Fäden seiner Figur 21 haben isodiametrische Zellen; in Figur 20 sind aber die beiden Erscheinungsformen nebenein- 

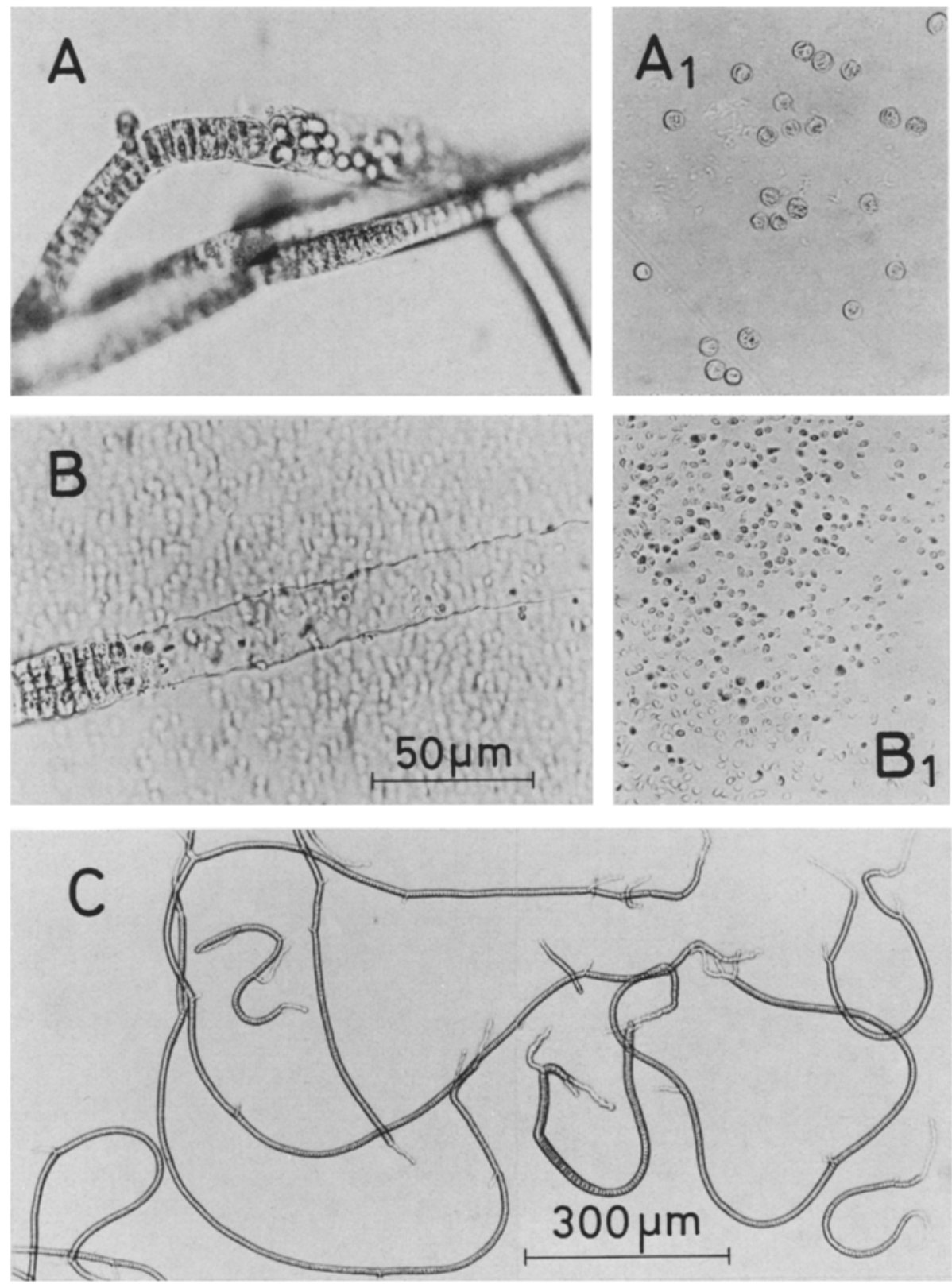

Abb. 5: Rosenvingiella polyrbiza. $A, A_{1}$ Teilweise entleertes weibliches Gametangium und Eier. $B, B_{1}$ Entleertes männliches Gametangium und Spermatozoiden. $C$ Fäden aus parthenogenetisch gekeimten Eiern 

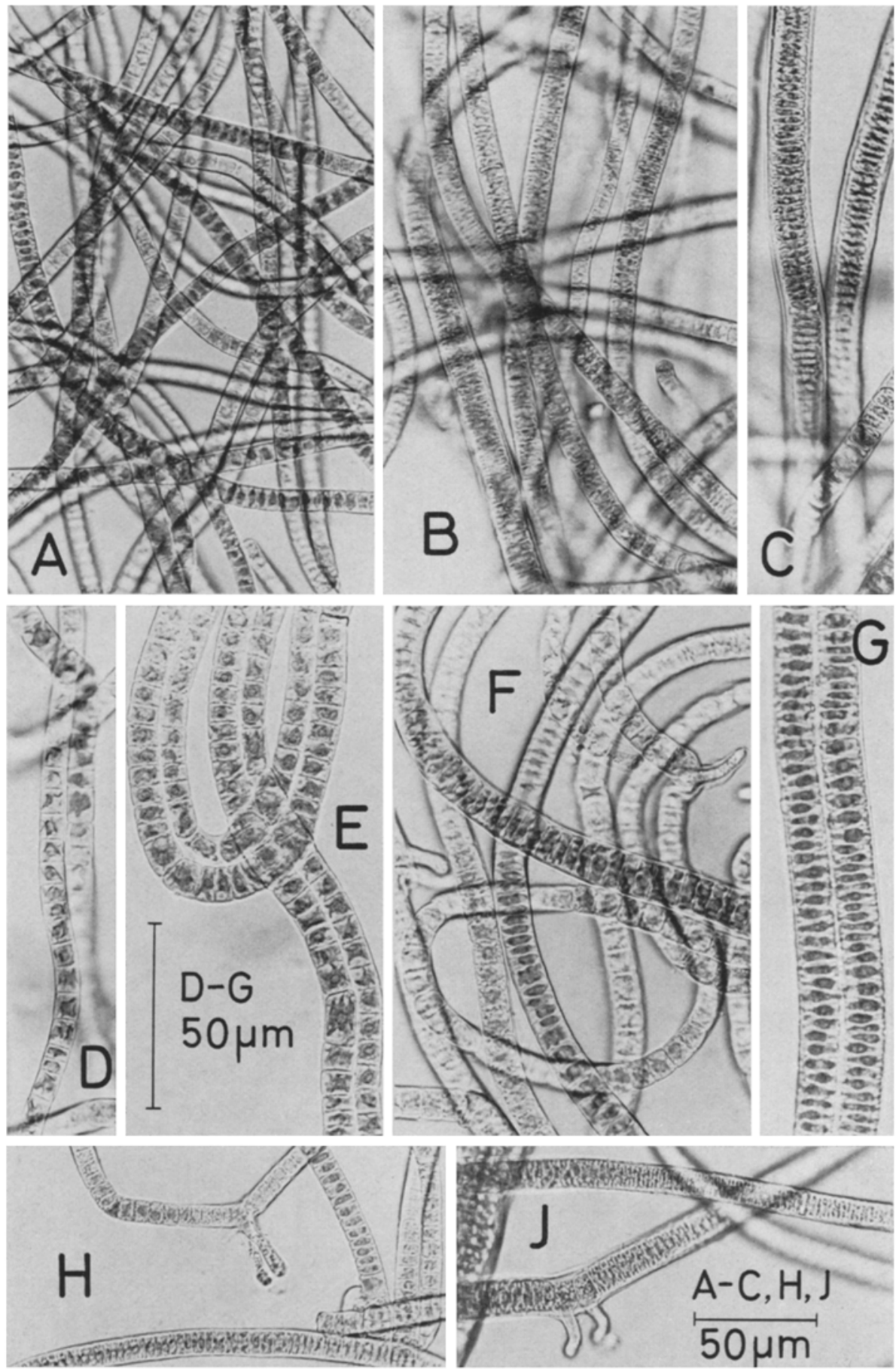

Abb. 6: Rosenvingiella polyrhiza. Variation der vegetativen Fäden 


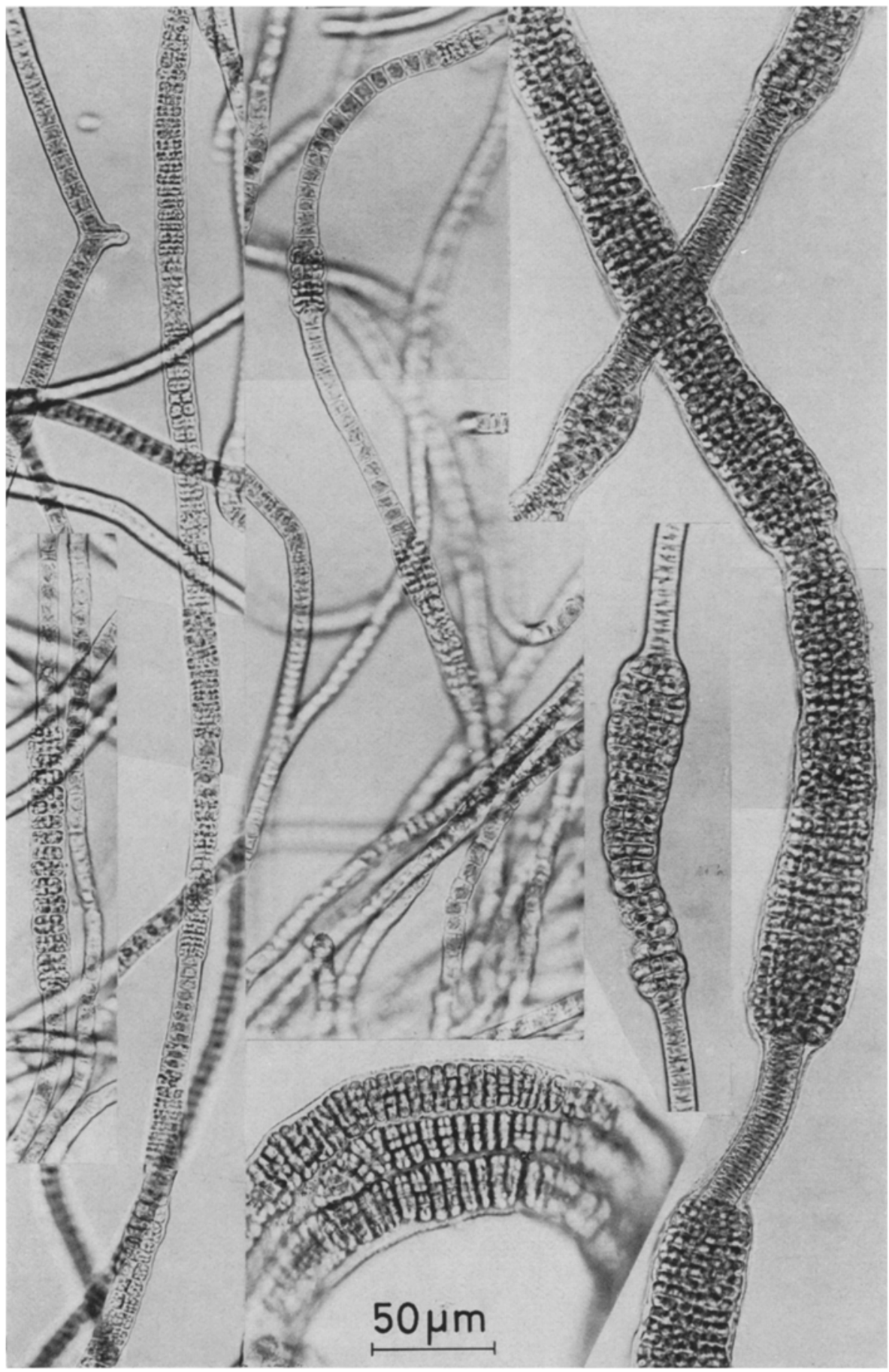

Abb. 7: Rosenvingiella polyrbiza. Variation der fertilen Fäden 
ander dargestellt: bei a und b die dünnfädige mit isodiametrischen, bei e eine breitere Form mit flachen Zellen, die dem von Rosenvinge dargestellten Typus entspricht.

Auch die Helgoländer Aufsammlungen enthalten im allgemeinen Fäden von unterschiedlicher Breite, wobei in den einzelnen Proben die dünnen oder die dickeren Fäden überwiegen können. Eine klare Beziehung zu den Standortfaktoren ließ sich dabei nicht erkennen, und in Kultur behielten die auf Einzelfäden zurückgehenden Stämme ihre Fadenbreite bei. Abbildung 6 zeigt das verschiedenartige Aussehen solcher Proben. Bei A liegt die Fadenbreite zwischen 7,5 und $9 \mu \mathrm{m}$, die ausgewachsenen Zellen sind isodiametrisch. Die Fäden bei B und C sind 11 bis 14 beziehungsweise bis $18 \mu \mathrm{m}$ breit; ihre Zellen sind scheibenförmig. Die Wechselbeziehung zwischen Fadenbreite und Zellenlänge kommt in der Auswahl von Fadenstücken bei D bis $G$ noch klarer zum Ausdruck.
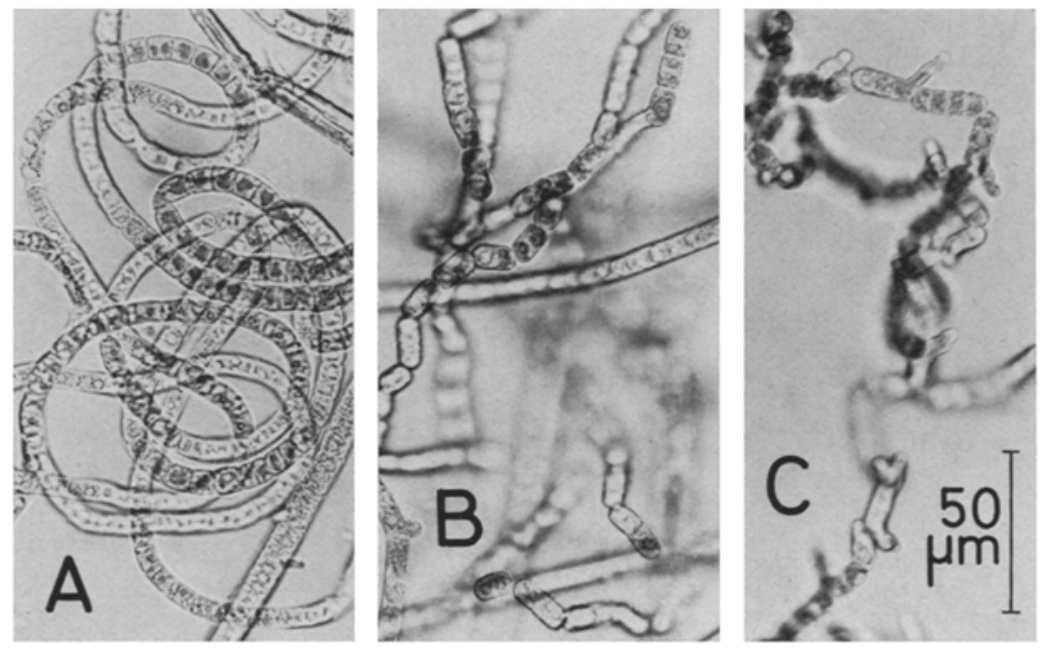

Abb. 8: Rosenvingiella polyrbiza. Fragmentierung

Mehrreihige, meist doppelte Fäden trifft man in allen Proben an (Abb. 6 E, G), gelegentlich sind auch bis zu fünf Fadenreihen miteinander vereinigt. Solche Doppelbildungen verbreitern sich nicht zu flächigen. Thalli, auch nicht nach monatelanger Kultur. Rhizoide kommen in den einzelnen Proben mit unterschiedlicher Häufigkeit vor, doch läßt sich auch hier keine klare Beziehung zu den Standortfaktoren erkennen. Meist entspringen sie paarweise benachbarten Zellen (Abb. $6 \mathrm{H}, \mathrm{J})^{*}$.

* Prasiola crispa - das Material wurde von P. Kornmann im Oktober 1973 im VillnößTal, Südtirol, gesammelt - bildete auch in Kultur niemals Rhizoide. Flädhige Thalli entstanden durch Verbreiterung mehrreihiger Fäden. Die beiden Taxa sind also klar gegeneinander abgegrenzt.

Auf Helgoland wurde Prasiola crispa bisher nur in einer Probe zwischen Rosenvingiella polyrhiza gefunden (17.3.1974, Anker auf dem Rasen vor Haus Wiking am Nordstrand). Es waren nur wenige kleine Thallusstücke, die sich durch ihre flächige Verbreiterung leicht von Rosenvingiella unterschieden. In Kultur verhielten sich die beiden Herkünfte ganz gleichartig. 
Die parenchymatischen Fadenabschnitte sehen sehr unterschiedlich aus (Abb. 7). Ein Faden kann auf einer längeren Strecke fertilisiert sein, wiederholt durch kurze Zonen vegetativer Zellen unterbrochen. Die Gametangien können aber auch als kurze Abschnitte in die Fäden eingeschaltet sein. Ihre Breite steht in direkter Beziehung zur Fadendicke; in den dünnfädigen Proben sind sie im allgemeinen schmal und kleingefächert und wegen ihrer blassen Färbung in den Präparaten nur bei stärkerer Vergrößerung zu erkennen. In den didkfädigen Proben sind die fertilen Fäden durch ihre Breite, die Größe der Fächer und die intensive Färbung der Zellen schon bei schwacher Vergrößerung nicht zu übersehen. Auch mehrreihige Fäden können fertilisiert werden.

Gelegentlich findet man dünne Fäden, deren Zellen an den Querwänden leicht tonnenförmig eingeschnürt sind (Abb. $8 \mathrm{~A}$ ). Auch WILLE bildet solche Fäden ab und deutet diese Zellen als Vermehrungsakineten (1901, Tafel I, Fig. 48a und 52). Sie sollen in kugeligen Sporangien kleine Aplanosporen bilden, die nach entsprechender Volumenzunahme zu monosiphonen Fäden auswachsen (Fig. 49-51). Man muß dieser Darstellung mit Vorbehalt begegnen. Sie gründet sich nicht auf Ergebnisse eines Kulturversuchs, sondern ist aus Beobachtungen an Naturmaterial kombiniert. Nach WILles Abbildungen wären die aus Aplanosporen hervorgegangenen Fäden etwa 1,5mal breiter als das Ursprungsmaterial, so daß ihre Zusammengehörigkeit zweifelhaft erscheint. WrLtes Beobachtungen lassen sich nicht mit Sicherheit deuten; vielleicht sind diese Aplanosporangien Stadien des Lebenszyklus von Prasiola calophylla, die häufig mit Rosenvingiella vergesellschaftet ist (siehe Abb. 13 und 14). Auch kann eine selbständige Protococcale im Naturmaterial nur schwer von solchen Aplanosporangien unterschieden werden. Wenn in unseren Kulturen die Fäden gelegentlich fragmentierten, so führte dies niemals zur Bildung von Vermehrungsakineten, sondern die Fragmente entwickelten sich unmittelbar zu selbständigen Fädchen (Abb. 8 B, C). An Naturmaterial wurde diese Erscheinung nicht beobachtet.

Neben den Gametangien, die während der Frühjahrsmonate, der Zeit der üppigsten Entfaltung von Rosenvingiella polyrbiza auftraten, konnten trotz eingehenden Suchens nur einmal Stadien gefunden werden, die auf eine ungeschlechtliche Vermehrung hinweisen könnten (Abb. 9 A, B). Sie wurden am 3. August 1973 an einer etwas feuchten und schattigen Stelle gesammelt, wo die Alge auch im Sommer noch verhältnismäßig gut entwickelt war. Auf meist nur kurzen Fadenabschnitten teilen sich die Zellen längs und werden kugelig. Diese Gebilde erinnern an Fäden mit Oogonien, jedoch sind die kugeligen Zellen erheblich dicker als Eier. Sie werden nicht als Sporen entleert, sondern keimen auch unter natürlichen Verhältnissen unmittelbar zu Fäden aus (Abb. $9 \mathrm{~B}$ ). In Kultur ließ sich ihre Entwicklung zu fädigen Keimlingen mit Rhizoiden leicht verfolgen; $A_{1}$ und $A_{2}$ sind Ausschnitte des bei $A$ dargestellten Fadens nach 7 beziehungsweise 11 Tagen. Es ist bemerkenswert, daß die im Frühjahr 1974 an dem gleichen Fundort gesammelten Proben reichlich parenchymatische Fadenabschnitte enthielten, doch lassen sich diese Beobachtungen nicht in einen Zusammenhang bringen.

Cysten - wir übernehmen diese Bezeichnung von KRISTIANSEN (1972) - wurden in einigen Aufsammlungen im August und September gefunden. Es sind einzelne oder in Reihen bis zu sechs in die Fäden eingeschaltete kugelige Zellen (Abb. 10). Ihre Rolle konnte nicht geklärt werden; im allgemeinen gingen sie in den Kulturen nach kurzer Zeit zugrunde, während die angrenzenden vegetativen Zellen sich lebhaft teilten. 


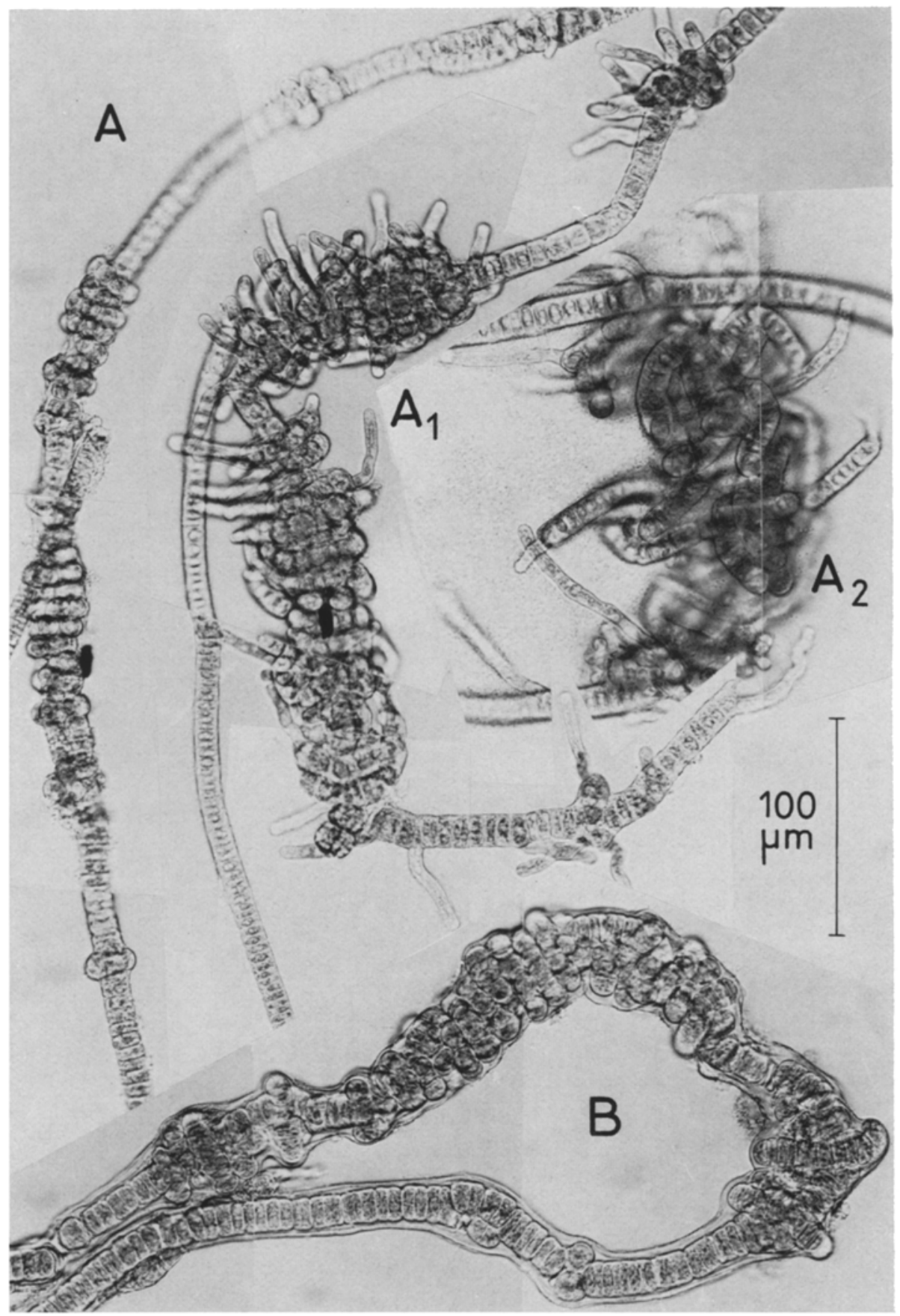

Abb. 9: Rosenvingiella polyrbiza. $A, B$ Ungeschlechtliche Vermehrung? $A_{1}, A_{2}$ Der gleiche Faden wie $A, 7$ beziehungsweise 11 Tage später mit Keimlingen 


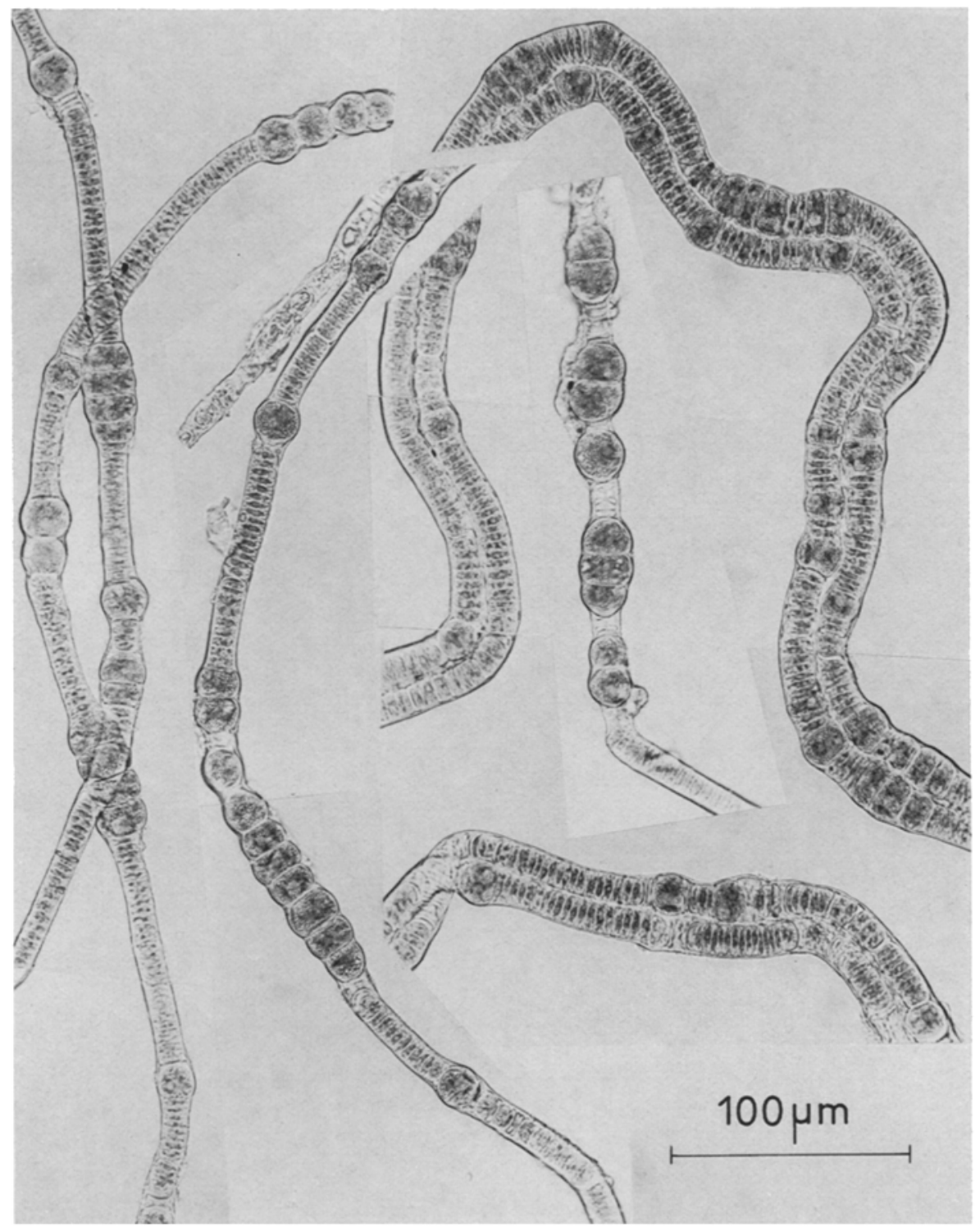

Abb. 10: Rosenvingiella polyrbiza. Fäden mit Cysten

KRISTIANSEN fand Cysten in ihrem nördlich von Kopenhagen gesammelten Material während des ganzen Jahres.

Mit großer Wahrscheinlichkeit wurden Erscheinungsformen von Rosenvingiella polyrbiza bereits von KützING (1852, Tab. phyc. II, Tafel 100) abgebildet. Schizogonium crispatum von Helgoland (Fig. 3) könnte ein Faden mit Cysten sein. Schizogo- 
nium copulatum (Fig. 4, = Lyngbya copulata HASSALL) entspricht durchaus einem Doppelfaden von Rosenvingiella, der dreireihig wird. Auch unter Schizogonium radicans KüTz. dürften sich Aufsammlungen verbergen, die zu Rosenvingiella polyrbiza gehören.

\section{Prasiola calopbylla (CARM. ex GREv.) MENEGH. \\ Vorkommen und Morphologie}

Die meisten auf Helgoland gesammelten Proben von Rosenvingiella polyrbiza enthielten eine kleine Prasiola, hellgrüne, 1 bis $3 \mathrm{~mm}$ hohe Büschel. Waren es mitunter auch nur einzelne Exemplare, so konnten sie doch oftmals reichlich vertreten sein und kamen schließlich an einzelnen Stellen auch in reinen Beständen vor. Die zwischen Rosenvingiella wachsenden Pflanzen waren im allgemeinen flach, junge Thalli schmal bis zungenförmig (Abb. $11 \mathrm{~A}, \mathrm{~B}$ ), ältere Exemplare breiter und nach dem Verlust der fertilen Spitze gerade oder unregelmäßig abgeschnitten (Abb. $11 \mathrm{C}$ ). Dagegen waren die am Fuße einer senkrechten Mauer aus einem reinen Bestand gesammelten Pflanzen korkzieherartig gewunden (Abb. $11 \mathrm{D}$ ). Trotz ihres so verschiedenen Aussehens haben diese Pflanzen in der gleichartigen Entwicklung ein untrügliches Merkmal ihrer $\mathrm{Zu}$ sammengehörigkeit.

Die klare Beschreibung und Abbildung von Prasiola calophylla bei Greville (1826, pl. 220) rechtfertigt die Zuordung unseres Materials zu dieser Art. Zwar erreichen die Büschel im allgemeinen nicht die von GREvILLE angegebene Länge von 2 bis 3 Linien, doch sind auch einzelne Exemplare dieser Größe zu finden. Das von CarmICHAEL gesammelte Typusmaterial wuchs "on a block of stone near the Clergyman"s house in the Island of Lismore", also keineswegs an einem marinen Standort. Auch auf Helgoland ist das Vorkommen nicht auf die Molen und Uferschutzbauten beschränkt; Prasiola calophylla kommt mit Rosenvingiella polyrbiza in großer Menge auf der zum Oberland führenden Haupttreppe und sogar mitten in der Siedlung auf dem Oberland vor.

Die Aufsicht auf den vegetativen Thallus zeigt nahezu quadratische, sehr regelmäßig in Längs- und Querreihen angeordnete Zellen, die bei der Fertilisierung kreuzweise geteilt werden (Abb.12 A, B, Abb. 15 C). Querschnitte durch lebend eingefrorenes Naturmaterial zeigen den einschichtigen vegetativen beziehungsweise den durch zwei aufeinanderfolgende Teilungsschritte vierschichtig gewordenen fertilen Thallus (Abb. $12 \mathrm{C}, \mathrm{D}$ ), bei $\mathrm{E}$ sind entsprechende Schnitte durch eine kultivierte Pflanze dargestellt. Aus jeder Zelle gehen mithin 16 Sporen hervor.

\section{Entwicklung}

In vielen Fällen war das am Standort gesammelte Material fertil; oftmals klebten noch Reste von Sporen am Rand des aufgelösten Thallus (Abb. 12 A, B). In Nährlösung übertragene Pflanzen werden im allgemeinen nach 10 bis 12 Tagen fertil; in einer ruhigstehenden Kultur bilden die entleerten Sporen ein dichtes, gallertiges Lager, 


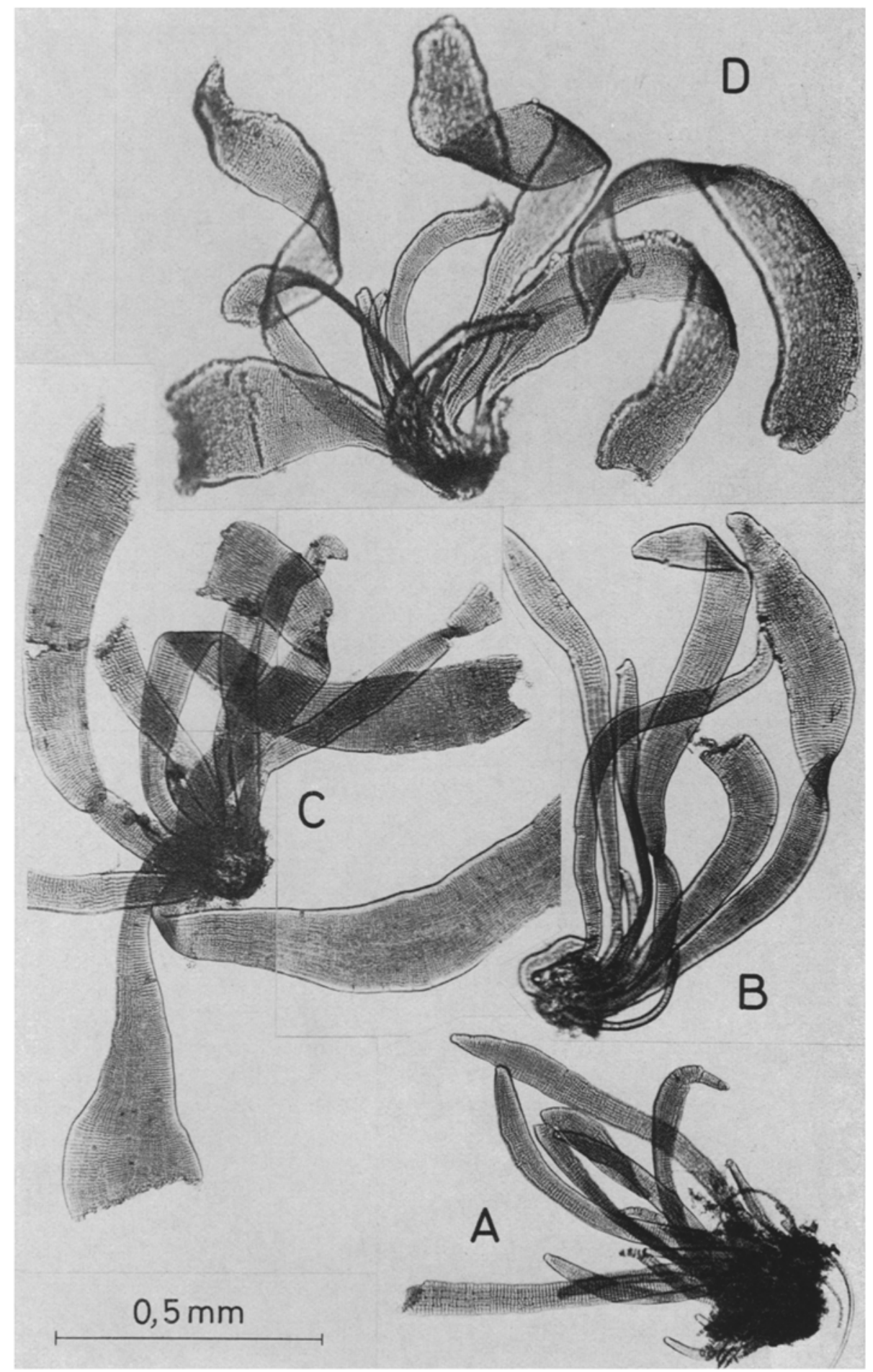

Abb. 11: Prasiola calophylla. $A, B$ Jüngere, $C$ ältere Pflanzen aus einer Watte von Rosenvin giella polyrbiza. $D$ Spiralig gewundene Pflanzen aus reinem Bestand 


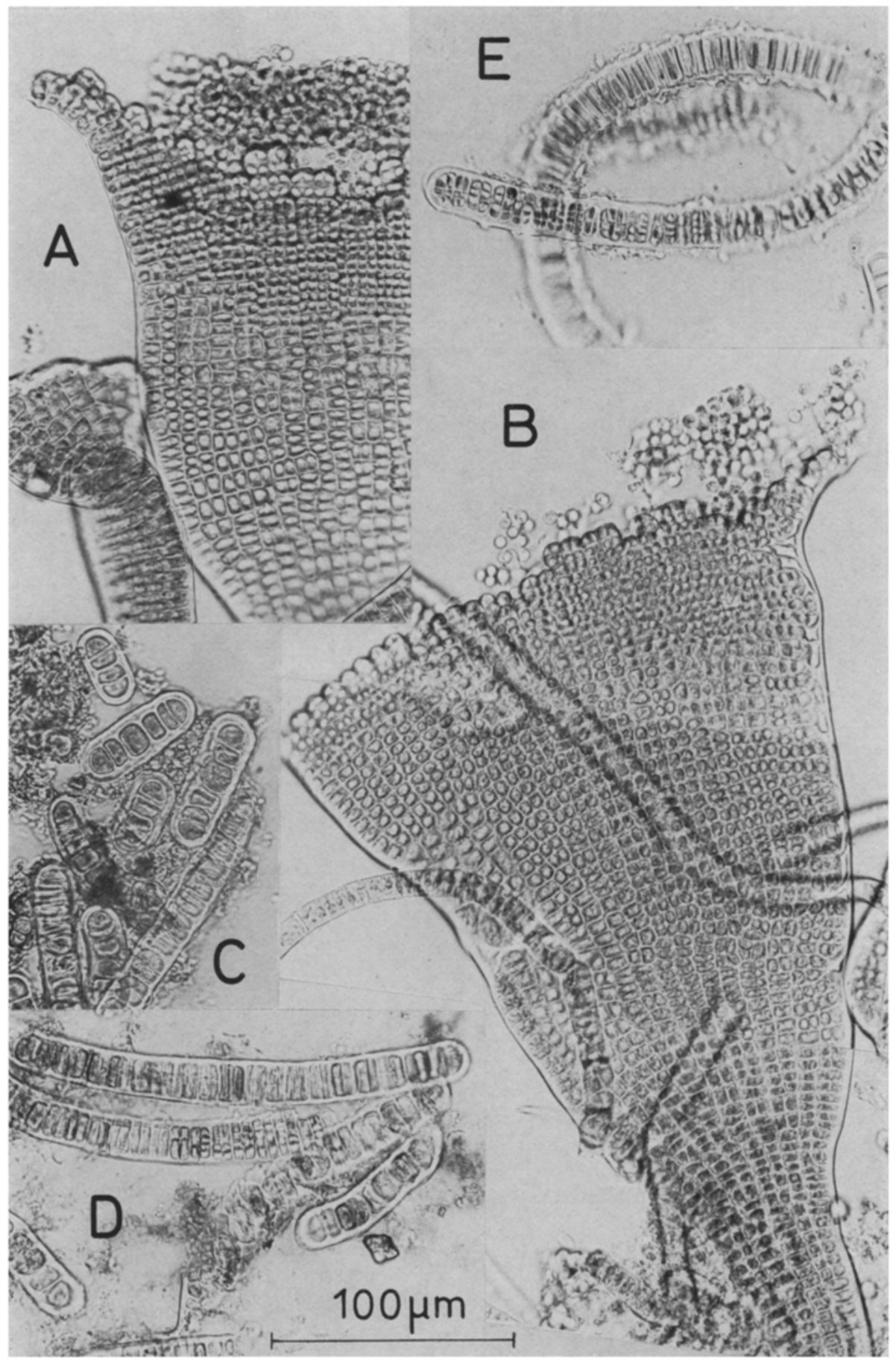

Abb. 12: Prasiola calopbylla. A, B Fertile Thalli mit anhaftenden Sporen. C, $D$ Querschnitte durch lebend eingefrorenes Natur-, $E$ durch Kulturmaterial 

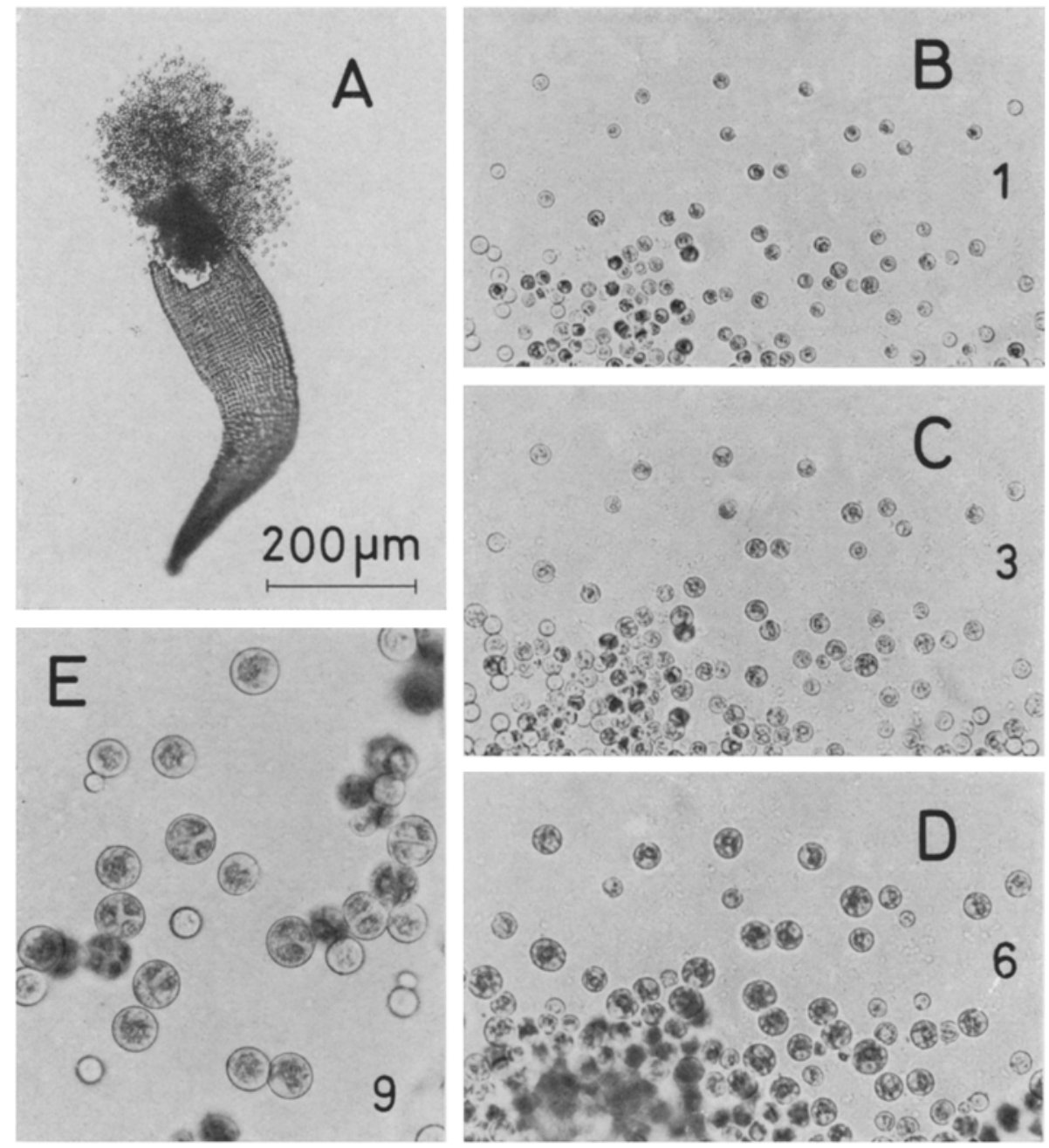

(a)
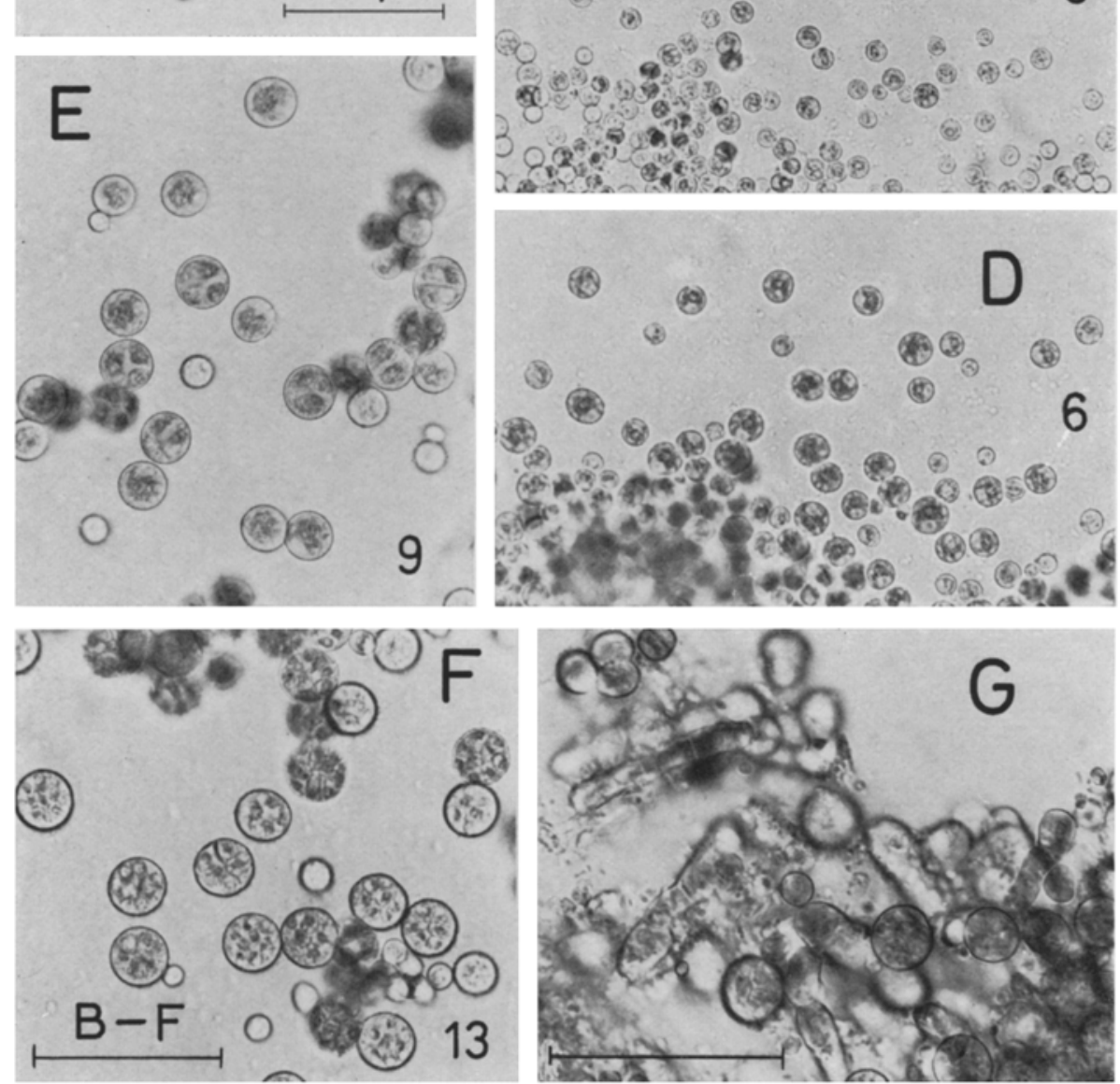

Abb. 13: Prasiola calopbylla. A In Nähriösung fertil gewordene Pflanze. $B-F$ Entwicklung der Sporen zu Aplanosporangien. Die eingetragenen Ziffern geben das jeweilige Alter der Kulturen an. $G$ Aus einem Präparat von natürlichem Material: zwischen den Aplanosporangien junge Prasiolopsis-Fädchen. Die Maßstäbe entsprechen jeweils $50 \mathrm{~km}$ 


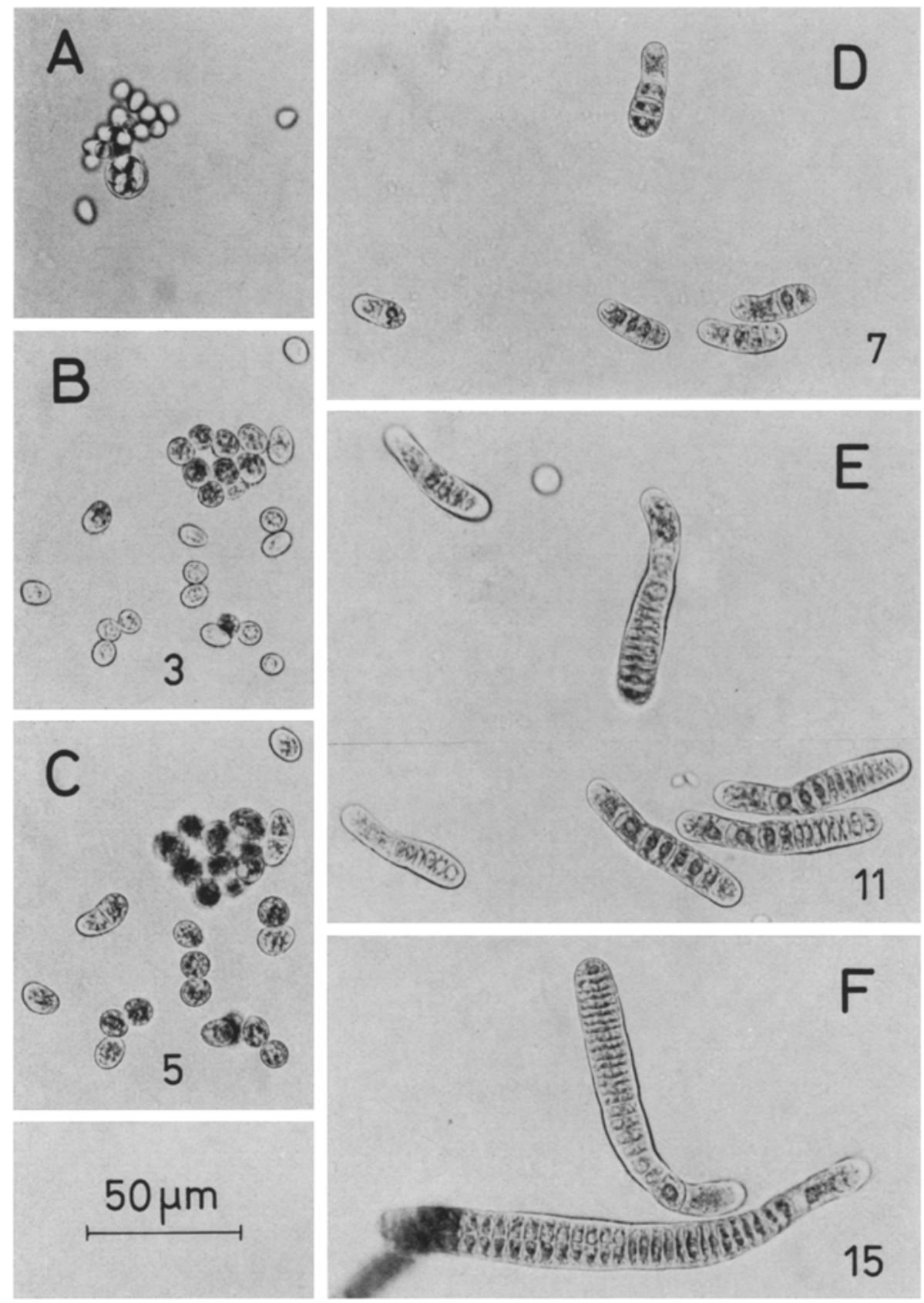

Abb. 14: Prasiola calophylla. Entwicklung der Aplanosporen zum flächigen Thallus. Die eingetragenen Ziffern geben das jeweilige Alter der Kulturen an 
aus dem sie sich nicht mit einer Kapillare aufsaugen lassen (Abb. 13 A). Die Aufteilung eines solchen Sporenhaufens in Gruppen mehrerer oder einzelne Sporen läßt sich nur durch mechanische Zerkleinerung und wiederholtes Aufsaugen und Ausblasen mit einer Kapillare erreichen.

Die frisch entleerten Sporen sind kugelig und 4 bis $5, \mu \mathrm{m}$ dick. Ihre ganze Entwicklung besteht in einer Volumenvergrößerung und - etwa vom achten Tage an - Aufteilung des Inhalts in Endosporen (Abb. 13 B-F). Nach 11 bis 12 Tagen waren unter den gewählten Kulturbedingungen die Kugeln bis zu $15 \mu$ m dick und polyedrisch gefeldert. Auch unter natürlichen Verhältnissen entwickeln sich die häufig am fertilen Thallusende haftengebliebenen Sporen in gleicher Weise. Abbildung 13 G zeigt ein solches Präparat mit reifen Aplanosporangien; die zwischen ihnen wachsenden Fädchen gehören zu Prasiolopsis (p. 128).

Die größten Sporangien - als solche kann man sie wohl bezeichnen - entleerten sich mitunter während der Beobachtung, vielleicht durch das helle Licht der Mikroskopierlampe ausgelöst (Abb. 14 A). Die Anzahl der in einem Sporangium gebildeten, meist leicht ovalen Aplanosporen liegt zwischen 8 und etwa 25, dementsprechend ist auch ihre Größe nicht einheitlich. Sie sind nicht in einer gallertigen Masse miteinander verklebt, lassen sich daher leicht in einer Kapillare auffangen und in Kultur nehmen.

Ihre Entwicklung zu einer neuen Prasiola-Generation zeigt keine Besonderheiten (Abb. 14). Nachdem die Sporen ihren Durchmesser etwas vergrößert haben, strecken sie sich zu einem monosiphonen Keimling. Schon im 3- bis 4zelligen Stadium, das nach 7 Tagen erreicht wird, hat sich der Keimling in eine gestreckte Rhizoid- und mehrere schmale Fadenzellen differenziert. An 14 Tagen alten und inzwischen $17 \mu \mathrm{m}$ breit gewordenen Fädchen erfolgen die ersten Längsteilungen. 5 Wochen alte Pflanzen sind etwa 5 bis $6 \mathrm{~mm}$ lang und nur etwa $0,1 \mathrm{~mm}$ breit, der Thallus ist leicht spiralig gedreht (Abb. $15 \mathrm{~A}$ ); ältere, nicht fertil gewordene Pflanzen sind korkzieherartig gewunden (Abb. 15 B). Die Fertilisierung kann am apikalen Ende oder auch in einer mittleren Zone der Alge beginnen. Wie bereits erwähnt, entstehen aus einer Thalluszelle durch kreuzweise antikline und doppelte perikline Teilungen jeweils 16 kleine Sporen. Bei ihrer Reife verquellen die Sporangienwände, und die Sporen werden wie bei dem Naturmaterial in einer gallertigen Masse entleert. Als hauchzartes Häutchen bleibt die den Thallus überziehende Außenmembran übrig (Abb. $15 \mathrm{C}$ ).

Die Lebensbedingungen der Alge an ihrem natürlichen Standort und in der Kultur sind überhaupt nicht vergleichbar. Nur gelegentlich von spritzendem Seewasser benetzt oder von Regenwasser überspült, gedeiht sie in Kultur dawernd untergetaucht ganz prächtig. Am besten wuchs sie in Süßwasser, das im Liter $50 \mathrm{ccm}$ Erdabkochung sowie Phosphat und Nitrat wie in Schreiberlösung enthielt. Die Raumtemperatur betrug $15^{\circ} \mathrm{C}$, die tägliche Beleuchtungsdauer 14 Stunden. Daß die so unterschiedlichen Wachstumsbedingungen in der Natur und in Kultur zu einer verschiedenen Ausprägung des Habitus führen, ist nicht verwunderlich.

\section{Allgemeine Betrachtungen}

Die Besonderheit im Lebenszyklus von Prasiola calopbylla ist die obligate Aufeinanderfolge verschieden gestalteter, durch Sporen sich vermehrender Generationen. 


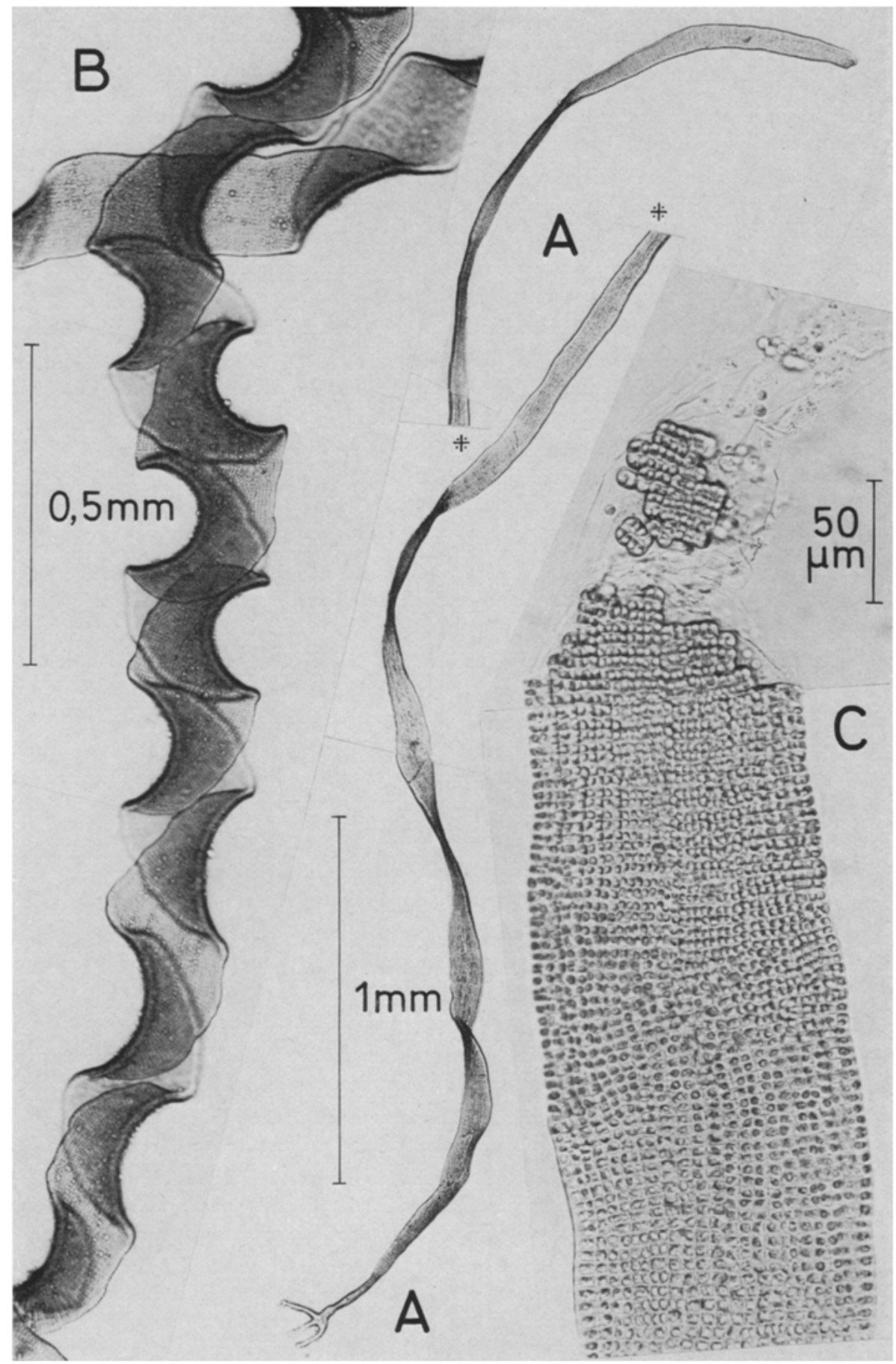

Abb. 15: Prasiola calopbylla. Aus Aplanosporen kultivierte Generation, $A 5$ Wochen alt, $B$ Ausschnitt aus einer nicht fertil gewordenen älteren Pflanze. $C$ Fertiles Thallusende 
Die auf dem flächigen Thallus gebildeten Sporen entwickeln sich zu einzelligen kugeligen Aplanosporangien, ein morphologischer Generationswechsel, der nicht mit einem Kernphasenwechsel verbunden ist. Einen ganz entsprechenden Zyklus hat Monostroma undulatum; Zoosporen werden auf dem flächigen Thallus und in den Cysten gebildet (Kornmann \& Sahling 1962, Tatewaki 1969).

Der hier für Prasiola calophylla im Kulturexperiment nachgewiesene Lebenszyklus entspricht weitgehend den Vorstellungen, zu denen WILLE (1901) auf Grund seiner Untersuchung natürlichen Materials über die Entwicklung von Prasiola gekommen war. Prasiola crispa forma submarina (= Rosenvingiella polyrbiza) sollte sich nach diesem Schema entwickeln, jedoch konnten wir weder an Naturmaterial noch in Kulturen entsprechende Beobachtungen machen (vgl p. 113). Selbst an Herbarmaterial von Prasiola crispa aus der Antarktis glaubte WILle (1902) diese Entwicklung erkennen zu können. Aus einzelnen am Rande des flächigen Thallus gebildeten Vermehrungszellen - die er aber an seinem Untersuchungsmaterial nicht gefunden hat - sollten Aplanosporangien entstehen, deren Sporen über ein einreihiges Hormidium-Stadium schließlich wieder zum flächigen Prasiola-Thallus führen sollten. War sich WILLE auch durchaus der Mängel bewußt, die einer Prüfung getrockneten Materials in Vergleich zu einer laufenden Beobachtung im Kulturversuch anhaften, so zögerte er einige Jahre später nicht, solche Kombinationen auf den Rang erwiesener Tatsachen zu erheben: "Bei Prasiola crispa (Lightf.) Menegh. forma submarina Wille habe ich früher (Studien' S. 17) nachgewiesen, daß die Vermehrungsakineten nicht immer direkt zu neuen Prasiola-Fäden auswuchsen, sondern zuweilen zuerst Aplanosporen bildeten, welche dann zu Fäden auswuchsen. Später habe ich (,Mittheilungen' S. 216, Taf. III, Fig. 16-19) eine ähnliche Entwicklung auch bei P. crispa (LightF.) Menegr. nachgewiesen. " (1906, S. 8.) Zu sehr von der Allgemeingültigkeit seiner Vorstellungen überzeugt, versucht WILLE (1906) dieses Schema auch auf die Entwicklung von Prasiola furfuracea zu übertragen. Deren Entwicklungsgang verläutt aber, wie im folgenden gezeigt wird, ohne Akineten und Aplanosporangien. Uberdies gehört zumindest ein Teil der von Wille abgebildeten Jugendstadien nicht zu Prasiola furfuracea, worauf bereits Brand (1925) hingewiesen hat. Näheres darüber unter Prasiolopsis (p. 128).

\section{Prasiola furfuracea (MERT.) MENEGH.}

\section{Vorkommen und Morphologie}

Seit Juli 1973 wurde Prasiola furfuracea auf einer nur wenige Quadratmeter großen Betonfläche der Mole des Nordosthafens gefunden. Die gleiche Stelle war im Winter und Frühjahr dicht von Rosenvingiella polyrbiza besiedelt, von der im Sommer nur noch spärliche Reste vorhanden waren. Die planmäßige Suche an vielen anderen Stellen blieb vergeblich.

An der Identität der Alge kann überhaupt kein Zweifel bestehen, ihr Habitus weist sie eindeutig als Prasiola furfuracea aus. Sie kann eigentlich nicht besser gekennzeichnet werden als durch die Beschreibung, die WAERN (1952, p. 42) gibt: „P. furfuracea is inter alia spoon-shaped or ear-shaped with the edges curved inwards and has 


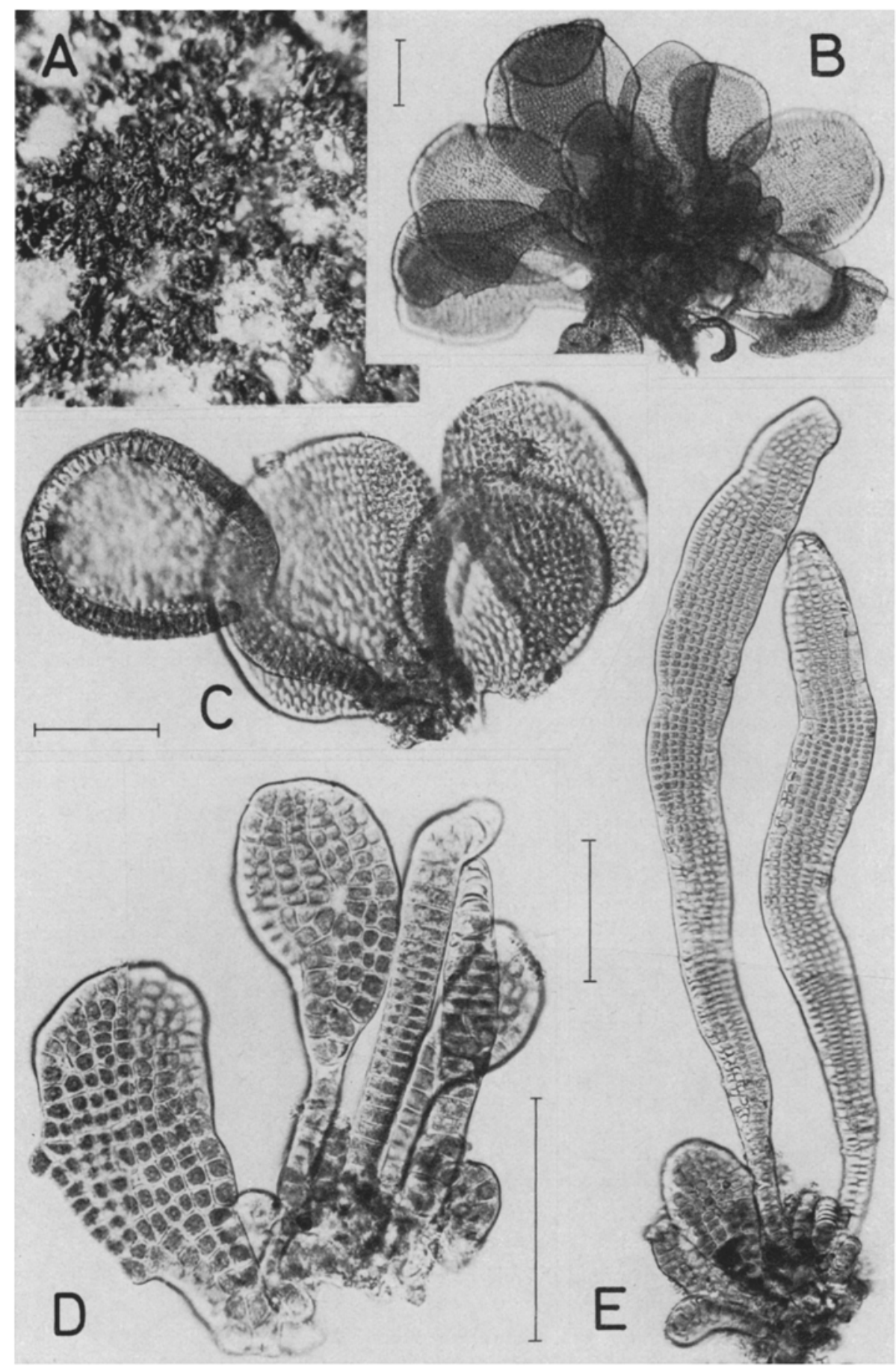

Abb. 16: Prasiola furfuracea. A Am Standort auf Beton, schwach vergrößßert. $B, C$ Büschel junger Pflanzen. $D, E$ Ebenso, jedoch mir Prasiola calopbylla zusammenwachsend, die sich in allen Entwicklungsstadien leicht unterscheiden läßt. Die Maßstäbe entsprechen jeweils $100 \mu \mathrm{m}$ 


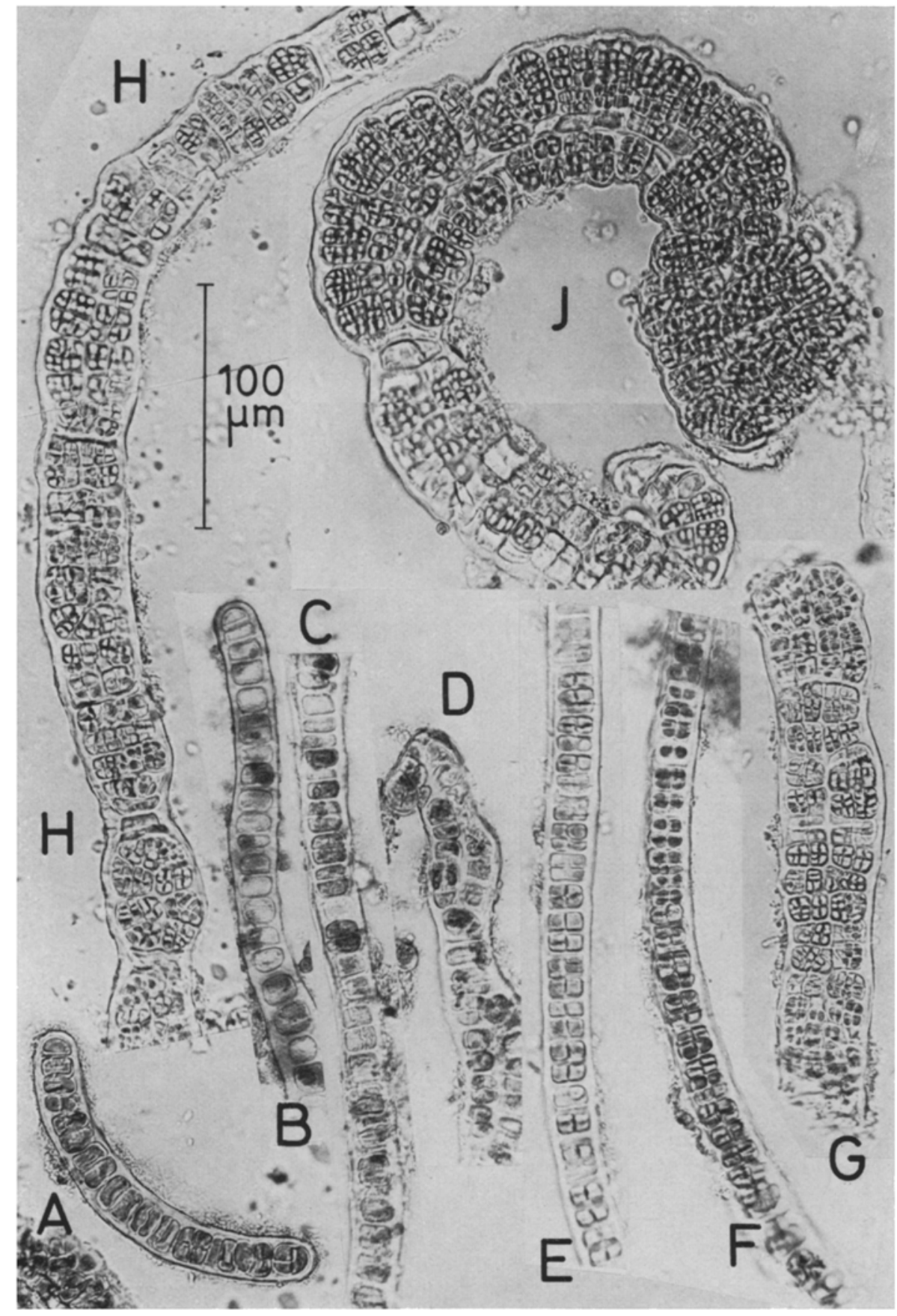

Abb. 17: Prasiola furfuracea. Querschnitte durch lebend eingefrorenes Material, frisch gesammelt $(A-D)$ bezichungsweise 10 Tage in Nährlösung gehältert $(E-J)$. A-C Vegetativer einschichtiger Thallus, $D$ doppelschichtiger Rand. $E, F$ Beginnende Fertilisierung. $G-J$ Fertiler Thallus beziehungsweise Randwulst 
very short stipes, or a few gathered rhizoids; sometimes the stipes was cut, as it were, into two or more, rhizoids; (in Prasiola stipitata no rhizoids, or splitting of the stipes were seen at all). The plant forms an extensive, darkgreen, granular layer consisting of numerous, densely agglomerated tufted individuals, generally few (2-6) in each tuft." Abbildung 16 A zeigt die Plänzchen an ihrem Standort nach Benetzung, Abbildung $18 \mathrm{C}$ den Habitus eines einzelnen Büschels. Durch ihre rundliche Form sind auch die jüngsten Stadien von Prasiola furfuracea genügend gekennzeichnet (Abb. 16 B, C); sie können stets sicher von Prasiola calopbylla unterschieden werden, mit der die Alge den Standort teilt. In Abbildung 16D lassen sich die beiden geraden, noch monosiphonen Fäden ohne weiteres als Prasiola calophylla erkennen; die bei $\mathrm{E}$ abgebildeten etwas größeren zungenförmigen Pflanzen verraten durch die sehr regelmäßig in Längs- und Querreihen geordneten und kleineren Zellen ihre Zugehörigkeit zu dieser Art. Dagegen bestimmt die rundliche, bei älteren Pflanzen auch ausgebuchtete Form des Thallus von Prasiola furfuracea zwangsläufig die weniger regelmäßige Anordnung der Zellen (Abb. 18 A). Eine Areolierung, wie sie JEssen (1848) darstellt und auch als systematisches Merkmal verwendet, ist an lebendem Material nicht zu erkennen. Querschnitte durch lebend eingefrorene Pflanzen zeigen den einschichtigen vegetativen Thallus, der aber am Rand auch unregelmäßig zweischichtig werden kann (Abb. 17 A-C, D).

Die im Juli und August gesammelten Pflanzen waren vegetativ; nichts deutete auf eine bevorstehende Fertilisierung hin. Jeweils wenige Pflänzchen wurden in drei verschiedenen Medien gehältert: Erdschreiberlösung, einer entsprechend mit Süßwasser bereiteten und einer ebensolchen mit doppelter Nährsalz- und Erddekoktmenge. Nur in der letzteren wurden nach 9 bis 12 Tagen mehrere Pflänzchen fertil und entleerten große Mengen sehr kleiner Sporen, die in einer gallertigen Masse verklebt den Schalenboden dicht bedeckten (Abb. $18 \mathrm{D}$ ). Nach der Entleerung bleibt eine hyaline Membran mit einzelnen Inseln noch unreifer Sporangien übrig, auch lassen Epiphyten auf der Außenhülle den Unriß des entleerten Thallus erkennen.

Die Fertilisierung beginnt mit einer Teilung der vegetativen Zellen in der Thallusebene, zugleich findet eine kreuzweise Aufteilung statt, so daß in der Umwandlungszone aus jeder vegetativen Zelle eine achtzellige Sporangienanlage entsteht (Abb. 17 E, F). Durch weitere horizontale Teilungen werden kleine Sporen gebildet, dabei erreicht der fertile Thallus etwa die doppelte Dicke des vegetativen (Abb. 17 G, H). Besonders dick und wulstig kann ein fertiler Thallusrand werden, der im vegetativen Zustand bereits zweischichtig war (Abb.17 J). Die Aufsicht auf eine fertile Thallusfäche zeigt die gegeneinander abgerundeten, unregelmäßig angeordneten Sporangien (Abb. 18 B). Ganz charakteristisch sind die durch ihre Abrundung zwangsläufig entstandenen "Zwickel".

Die Fertilisierung vegetativer Pflanzen konnte in der beschriebenen Weise wiederholt im Experiment erreicht werden. Nur einmal, offenbar durch besondere Witterungsverhältnisse bedingt, wurden auch fertile Pflanzen am natürlichen Standort gesammelt. Nach wochenlanger Trockenheit regnete es in der zweiten Septemberhälfte mehrfach ausgiebig. Etwa eine Woche später waren viele Pflanzen fertil (Abb. 18 B). 

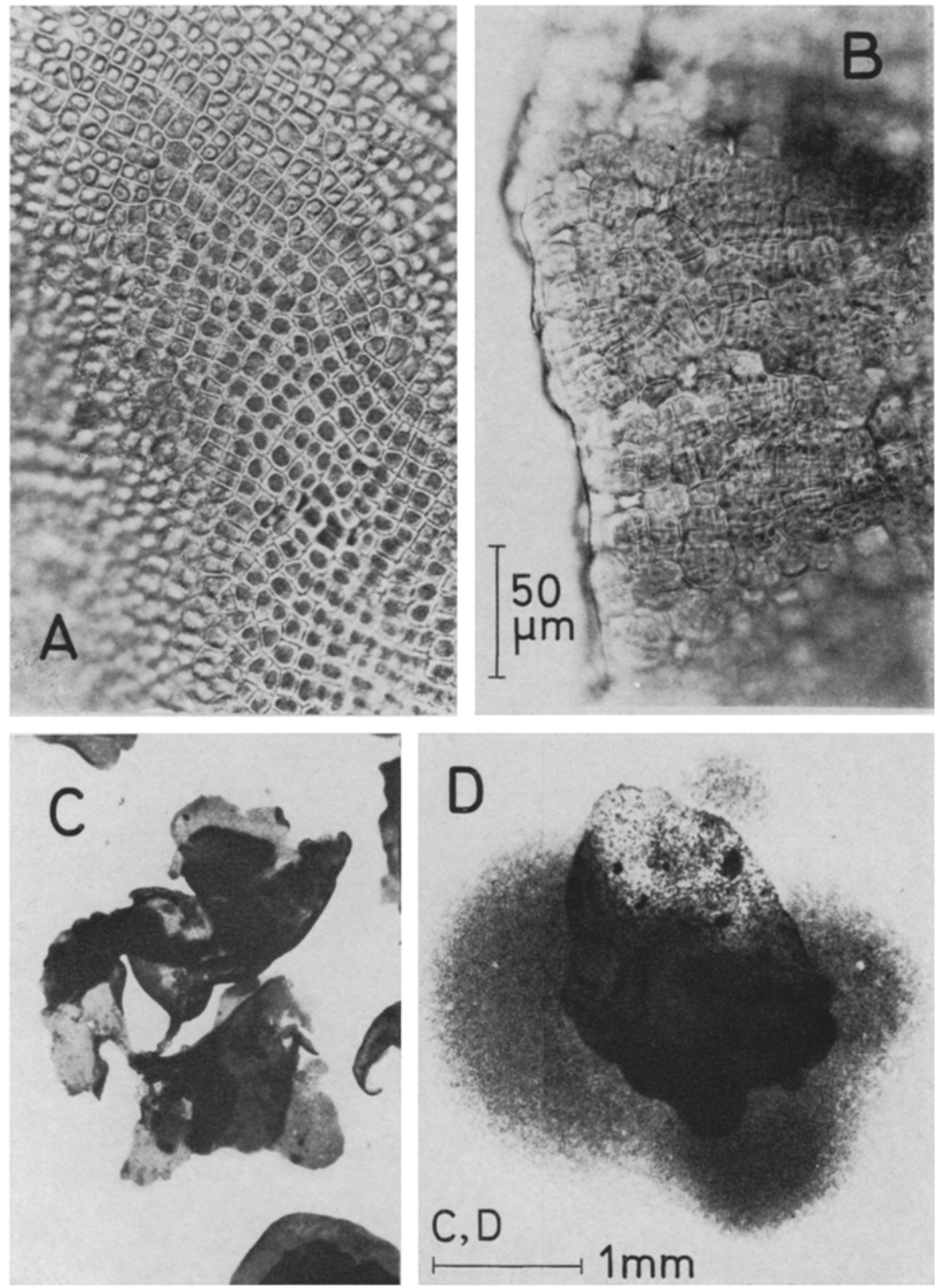

Abb. 18: Prasiola furfuracea. $A, B$ Aufsicht auf den vegetativen und fertilen Thallus. $C$ Büschel vegetativer Pflanzen. $D$ in Nährlösung fertil gewordener Thallus mit entleerter Sporenmasse 


\section{Entwicklung}

Die nach der Entleerung 4 bis $5 \mu \mathrm{m}$ dicken Sporen (Abb. 19 A) beginnen erst nach etwa 3 Tagen merklich zu wachsen, haben aber nach einer Woche ihren Durchmesser auf 8 bis $10 \mu \mathrm{m}$ vergrößert. Eine 10 Tage alte Kultur enthält schon viele oval gestreckte Stadien, einzelne mit einer Querwand. 18 Tage alte Keimlinge sind bereits in den flächig werdenden Thallus und das Rhizoid differenziert, das doppelt sein kann. Nach lebhaftem Wachstum zeigen die 4 Wochen alten Pflänzchen das für Prasiola furfuracea typische Zellmuster (Abb. $19 \mathrm{E}$ ). Thalli mit einem glatten Rand sind in den Kulturen selten, im allgemeinen wächst der Rand zu kurzen, gedrungenen Zellreihen aus (F). Immerhin bilden sich aber auch typische flächige oder schließlich muschelförmige Thalli aus $(\mathrm{G}, \mathrm{H})$.

Reinkulturen konnten durch mechanische Zerkleinerung der ausgetretenen Sporenmasse (Aufsaugen und Versprühen mit einer Kapillare) und Aufzucht in einer sehr dünnen Kultur erhalten werden, aus der die Keimlinge gegebenenfalls noch einmal isoliert werden mußten. Dauernd in Nährlösung untergetaucht wachsend, wurden die Pflänzchen von der 9. Woche an in der vorher beschriebenen Weise fertil. Eine andere Art der Fortpflanzung wurde nicht beobachtet.

Die Fertilisierung von Prasiola furfuracea ist offenbar noch niemals beschrieben und auch die Entleerung der Sporen noch nicht beobachtet worden. IMHÄUSER (1889), der zwar die Thallusentwicklung nach seinem in Marburg gesammelten Naturmaterial richtig wiedergibt, hat anscheinend die Fertilisierung nicht erkannt, doch deuten seine Abbildungen 57 und 68 auf reife Pflanzen hin. WILLES Untersuchung (1906) „Über die Entwidklung von Prasiola furfuracea (FL. D.) MENEGH. " kann hier unberücksichtigt bleiben; zweifellos enthielten seine am Standort entnommenen Proben Entwicklungsstadien verschiedener Arten. Schon Brand (1925, p. 339) äußert Bedenken, ob überhaupt eines der von WILle abgebildeten Entwicklungsstadien zu Prasiola furfuracea gehört. Keine der Zellflächen hat einen Stiel, der in dem von uns gesammelten Naturmaterial selbst bei ganz jungen Stadien nur selten fehlt. Eine „abweichende Entwicklung des jungen Thallus" (WrLe 1906, S. 37), wenigzellige, aber bereits fertile Fäden könnten sehr wohl als Prasiolopsis gedeutet werden; die „Vermehrungsakineten, welche sich zu Aplanosporangien umbilden“ (Tafel I, 15-23), könnten Stadien aus dem Entwicklungszyklus von Prasiola calopbylla sein. $\mathrm{Da}$ alle diese Arten mit Rosenvingiella polyrhiza, vielen einzelligen Grünalgen sowie Cyanophyceen vermischt vorkommen, sind Reinkulturen mit wohldefiniertem Ausgangsmaterial Voraussetzung für gesicherte Ergebnisse.

\section{Prasiolopsis ramosa VISCHER}

Die Gattung Prasiolopsis wurde von Vischer (1953) aus dem Pleurococcus-Komplex abgetrennt. Unter dem Namen Pleurococcus vulgaris Menegh. non Naegeli stellte CHOdAT (1909) die Alge in ausgezeichneten Abbildungen dar. Brand (1925) erkannte sie in einigen der von WLLE (1906) abgebildeten Entwicklungsstadien von Prasiola furfuracea. 


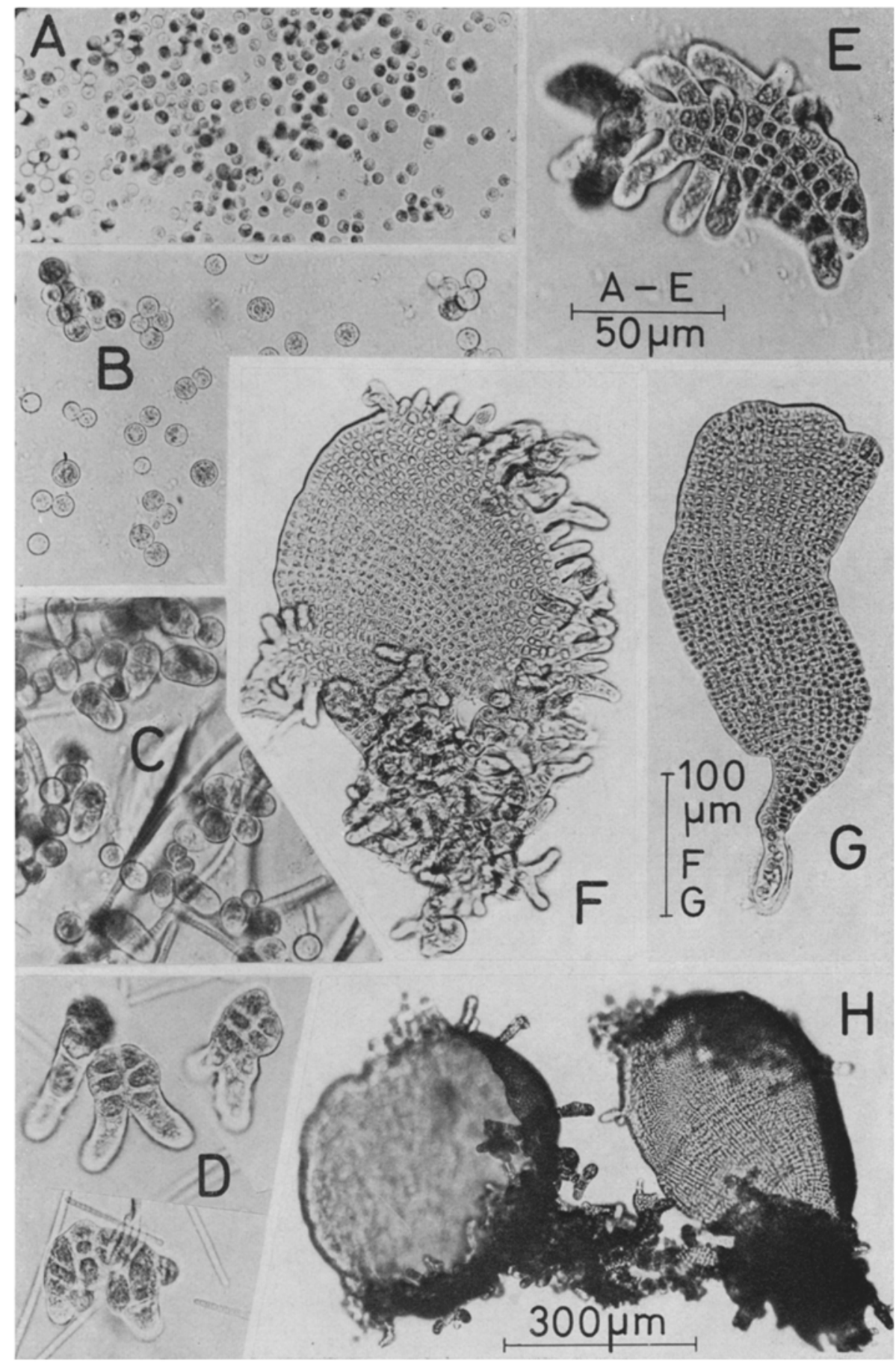

Abb. 19: Prasiola furfuracea. Entwicklung in Kultur. A Frisch entleerte Sporen. B-E Keimlinge im Alter von $7,12,18$ und 28 Tagen. $F, G$ Pflänzchen 7 und 8 Wochen alt. $H$ Muschelförmige, 9 Wochen alte Pflänzchen 

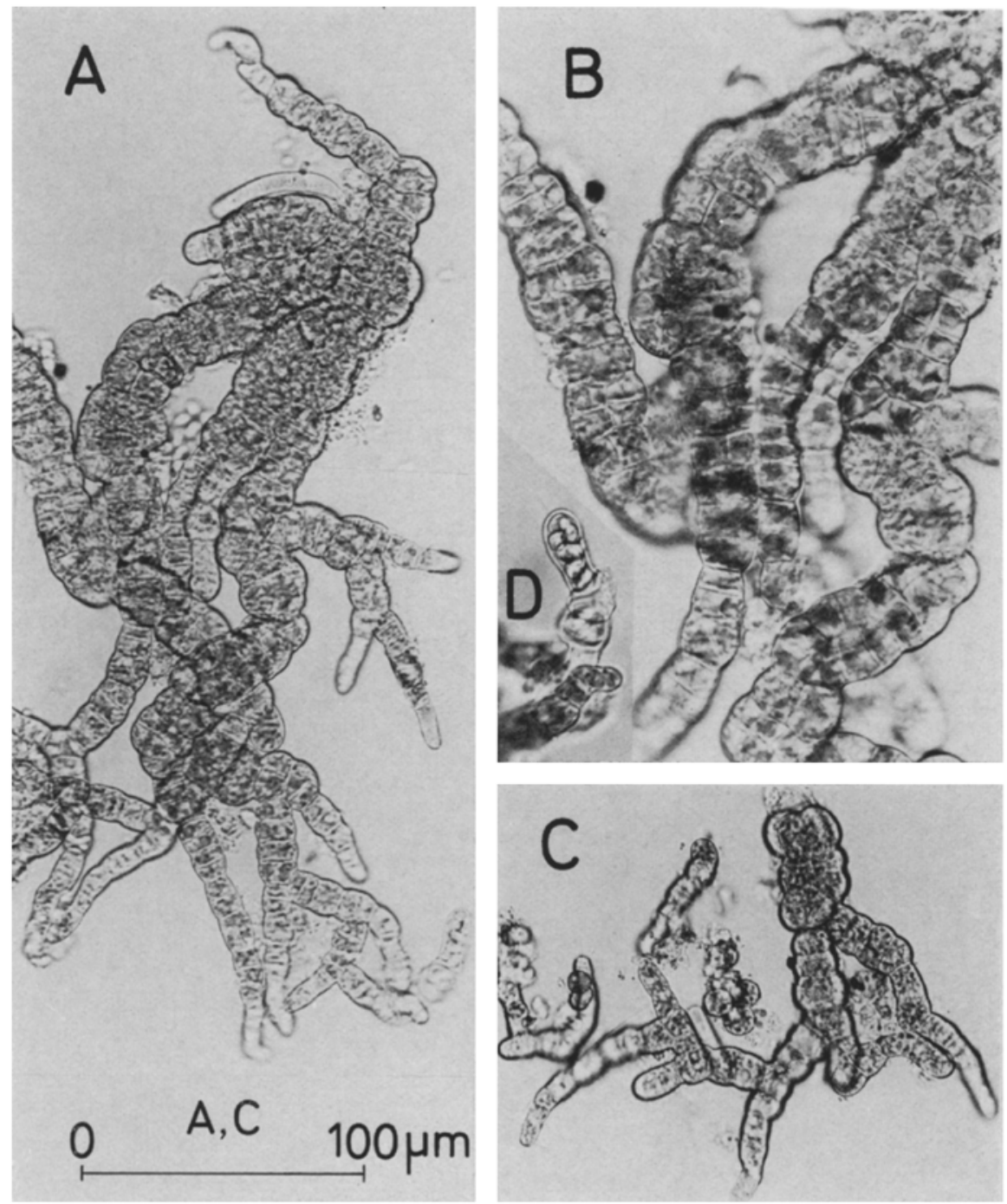

Abb. 20: Prasiolopsis ramosa. Naturmaterial. $B$ und $D$ sind Ausschnitte aus $A$

Nur selten findet man im natürlichen Material Prasiolopsis ramosa in so großen und wohlentwickelten Exemplaren wie in Abbildung $20 \mathrm{~A}$. Ihr verzweigter "parenchymatischer" Thallus gleicht völlig den von VIscher (1953, Abb. 10, 7-10) dargestellten Luftsprossen. Ein Fadenende mit entleerten Zellen und einem reifen Aplanosporangium zeigt Abbildung $20 \mathrm{D}$.

Prasiolopsis ramosa kommt überall auf den Molen zusammen mit Rosenvingiella polyrbiza und den sie begleitenden Prasiola-Arten vor. Mit großer Regelmäßigkeit stellt sie sich in Rohkulturen dieser Algen ein. Bei einiger Vertrautheit mit dem Objekt 

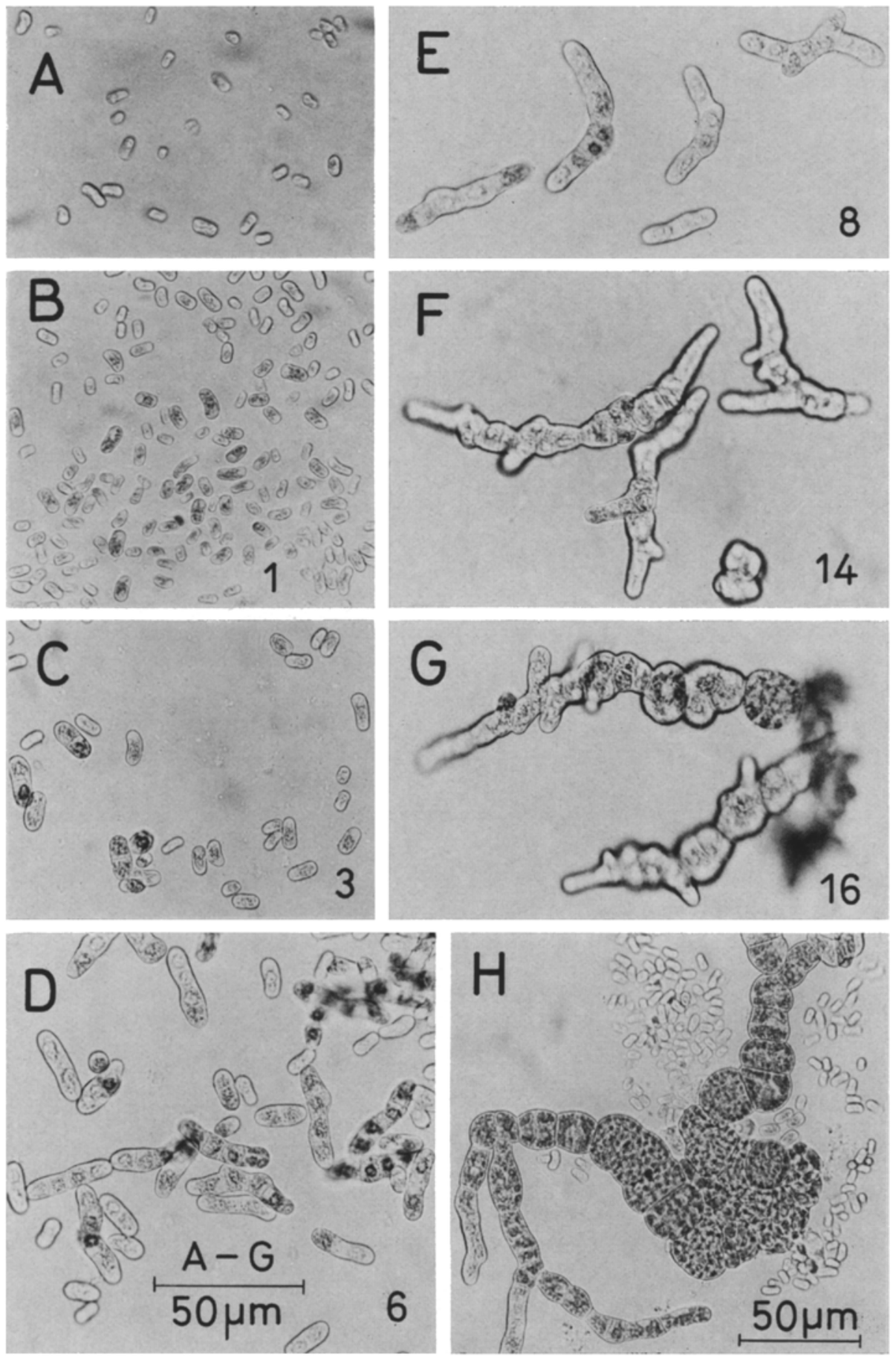

Abb. 21: Prasiolopsis ramosa. A-G Entwicklung der Sporen zu fertilen Thalli während 16 Tagen; die eingetragenen Ziffern geben das Alter der Kultur an. $H$ Fertiler Thallus, unter dem Deckglas etwas gepreßt 
lassen sich auch schon ganz junge Stadien sicher erkennen, wie zum Beispiel in dem der Abbildung $13 \mathrm{G}$ zugrundeliegenden Präparat. Die Prasiolopsis-Fädchen liegen hier zwischen den Aplanosporangien von Prasiola calopbylla, sie entsprechen etwa 6 Tage alten kultivierten Stadien (Abb. 21 D).

Der in einer Süßwasser-Nährlösung gut wachsende Thallus besteht aus unregelmäßig verzweigten Fäden, 5 Wochen alte Pflänzchen gleichen völlig dem Ausgangsmaterial. Vor ihrer Fertilisierung vergrößern sich die Fadenzellen und kugeln sich ab, ihr Inhalt wird in zahlreiche längliche Sporen recht unterschiedlicher Größe aufgeteilt $(\mathrm{Abb} .21 \mathrm{H})$. Sie wachsen schnell heran, und in einer drei Tage alten Kultur sind die größten Keimlinge schon zweizellig. Adht Tage alte Pflänzchen beginnen sich bereits zu verzweigen, kurz darauf kann man die ersten Längswände finden. Schon nach 16 Tagen können die Thalli fertil werden.

\section{ZUSAMMENFASSUNG}

1. Die als Gayella-Stadium von Rosenvingiella polyrbiza bezeichneten parenchymatischen Fadenabschnitte sind Gametangien: weibliche mit Eiern, männliche mit zweigeißeligen Spermatozoiden. Die vegetativen Fäden sind monosiphon; gelegentlich anzutreffende mehrreihige Bänder verbreitern sich nicht $z u$ einem flächenförmigen Thallus.

2. Rosenvingiella constricta, erstmalig bei Helgoland gefunden, ist ein diözischer Haplont. Die Reduktionsteilung erfolgt in der keimenden Zygote, aus ihr entstehen je zwei männliche und weibliche Gametophyten.

3. Prasiola calopbylla, häufig zusammen mit Rosenvingiella polyrbiza vorkommend, hat obligaten Wechsel ungleicher Generationen mit ungeschlechtlichen Sporen. Aus den Sporen des flächigen Thallus entstehen einzellige kugelige Aplanosporangien.

4. Prasiola furfuracea vermehrt sich unmittelbar durch Sporen, in die der größte Teil des Thallus aufgelöst wird.

5. In Rohkulturen von Rosenvingiella polyrbiza kommt neben zahlreichen anderen Grünalgen fast regelmäßig Prasiolopsis ramosa vor. Einzige Vermehrung durch Sporen.

Danksagung: Frau M. Kanje gebührt unser Dank für die Anfertigung der Gefriermikrotom-Schnitte.

\section{ZITIERTE LITERATUR}

Børgesen, F., 1902. The marine algae of the Faeröes. Bojesen, Copenhagen, 2, 339-352.

Brand, F., 1925. Analyse der aerophilen Grünalgenanflüge, insbesondere der proto-pleurococcoiden Formen. Arch. Protistenk. 52, 265-355.

Bravo, L. M., 1962. A contribution to knowledge of the life history of Prasiola meridionalis. Phycologia 2, 17-23.

- 1965. Studies on the life history of Prasiola meridionalis. Phycologia 3, 177-194.

Chodat, R., 1909. Etude critique et expérimentale sur le polymorphisme des algues. Genf, 165 pp. 
Cole, K. \& Arintobi, S., 1963. The life cycle of Prasiola meridionalis Setchell and Gardner. Can. J. Bot. 41, 661-668.

Frtedmann, I., 1959. Structure, life-history, and sex determination of Prasiola stipitata Suhr. Ann. Bot. (N.S.) 23, 571-594.

- 1960. Gametes, fertilization and zygote development in Prasiola stipitata Surr. I. Light Microscopy. Nova Hedwigia 1, 333-344.

- 1969. Geographic and environmental factors controlling life history and morphology in Prasiola stipitata SuHr. Óst. bot. Z. 116, 203-225.

Fujiyama, T., 1955. On the life-history of Prasiola japonica Yatabe. J. Fac. Fish. Anim. Husb. Hiroshima Univ. 1, 15-37.

Gardner, N. L., 1917. New Pacific coast marine algae I. Univ. Calif. Publs Bot. 6, 377-416.

Greville, R., 1826. Scottish Cryptogamic Flora. Edinburgh, 4.

IMHÄUSER, L., 1889. Entwicklungsgeschichte und Formenkreis von Prasiola. Flora, Jena, 72, 233-290.

JESSEN, C. F. G., 1848. Prasiolae generis algarum monographia. Diss. Kiel, 20 pp.

Kornmann, P. \& Sahling, P.-H., 1962. Zur Taxonomie und Entwidklung der MonostromaArten von Helgoland. Helgoländer wiss. Meeresunters. 8, 302-320.

Kristiansen, A., 1972. A seasonal study of the marine algal vegetation in Tuborg Harbour, the Sound, Denmark. Bot. Tidsskr. 67, 201-244.

KÜtzING, F. T., 1852. Tabulae phycologicae. Köhne [in Komm.], Nordhausen, 2, 1-37.

Rosenvinge, L. K., 1893. Gronlands Havalger. Meddr Grønland 3, 763-881.

Tatewaki, M., 1969. Culture studies on the life history of some species of the genus Monostroma. Scient. Pap. Inst. algol. Res. Hokkaido Univ. 6, 1-56.

VISCHER, W., 1953. Uber primitivste Landpflanzen. Ber. schweiz. bot. Ges. 63, 169-193.

WAERN, M., 1952. Rocky-shore algae in the Öregrund archipelago. Acta phytogeogr. suec. 30, $1-298$.

WILLE, N., 1901. Studien über Chlorophyceen I-VII. III. Eine submarine Form von Prasiola crispa (Lightr.). K. norske Vidensk. Selsk. Skr. 1900. (6), 1-46.

- 1902. Mittheilungen über einige von C. E. BorCHGREvinK auf dem antarktischen Festlande gesammelte Pflanzen. III Antarktische Algen. 1. Prasiola crispa (Lightr.) Menegh. Nyt Mag. Naturvid. 40, 209-221.

- 1906. Algologische Untersuchungen an der Biologischen Station in Dronthein I-VII. I. Uber die Entwicklung von Prasiola furfuracea (Fu. D.) Menegh. K. norske Videns'k. Selsk. Skr. $1906(3), 4-38$.

Anschrift des erstgenannten Autors: Dr. Dr. h. c. P. Kornmann

Biologische Anstalt Helgoland (Meeresstation)

2192 Helgoland

Bundesrepublik Deutschland 